\title{
On the thermodynamic and kinetic aspects of immersion ice nucleation
}

\author{
Donifan Barahona \\ NASA Goddard Space Flight Center, Greenbelt, MD, USA \\ Correspondence: Donifan Barahona (donifan.o.barahona@nasa.gov)
}

Received: 3 November 2017 - Discussion started: 30 November 2017

Revised: 5 October 2018 - Accepted: 19 October 2018 - Published: 4 December 2018

\begin{abstract}
Heterogeneous ice nucleation initiated by particles immersed within droplets is likely the main pathway of ice formation in the atmosphere. Theoretical models commonly used to describe this process assume that it mimics ice formation from the vapor, neglecting interactions unique to the liquid phase. This work introduces a new approach that accounts for such interactions by linking the ability of particles to promote ice formation to the modification of the properties of water near the particle-liquid interface. It is shown that the same mechanism that lowers the thermodynamic barrier for ice nucleation also tends to decrease the mobility of water molecules, hence the ice-liquid interfacial flux. Heterogeneous ice nucleation in the liquid phase is thus determined by the competition between thermodynamic and kinetic constraints to the formation and propagation of ice. At the limit, ice nucleation may be mediated by kinetic factors instead of the nucleation work. This new ice nucleation regime is termed spinodal ice nucleation. The comparison of predicted nucleation rates against published data suggests that some materials of atmospheric relevance may nucleate ice in this regime.
\end{abstract}

\section{Introduction}

Ice nucleation in cloud droplets and aerosol particles leads to cloud formation and glaciation at low temperatures. It is often initiated by certain aerosol species known as ice nucleating particles (INPs) (DeMott et al., 2003; Cziczo et al., 2013; Barahona et al., 2017). These include dust, biological particles, metals, effloresced sulfate and sea salt, organic material and soot (Murray et al., 2012; Hoose and Möhler, 2012). Background INP concentrations may be influenced by aerosol emissions (Lohmann and Feichter, 2005), altering the formation and evolution of ice clouds and leading to an indirect effect on climate. The assessment of the role of INPs in climate is challenging due to the complexity of the atmospheric processes involving ice and the limited understanding of the ice nucleation mechanism of INPs (Myhre et al., 2013). Ice formation promoted by a particle completely immersed within the liquid phase, referred as "immersion freezing", is likely the most common cloud glaciation pathway in the atmosphere (DeMott et al., 2003; Wiacek et al., 2010). Immersion freezing is involved in the initiation of precipitation and determines, to a large extent, the phase partitioning in convective clouds (Diehl and Wurzler, 2004; Wiacek et al., 2010; Lance et al., 2011; Murray et al., 2012).

The accurate representation of immersion ice nucleation is critical for the correct modeling of cloud processes in the atmosphere (Hoose and Möhler, 2012; Murray et al., 2012; Tan et al., 2016). Field campaign data have been used to develop empirical formulations relating the INP concentration to the cloud temperature, $T$, and saturation ratio, $S_{\mathrm{i}}$ (e.g, Bigg, 1953; Fletcher, 1959; Meyers et al., 1992), and more recently to the ambient aerosol size and composition (e.g., DeMott et al., 2010; Niemand et al., 2012; Phillips et al., 2013). Empirical formulations provide a simple way to parameterize ice nucleation in atmospheric models (e.g., Gettelman et al., 2012; Barahona et al., 2014). However they may not be valid outside the conditions used in their development, particularly as different experimental techniques may result in a wide range of measured ice nucleation efficiencies (Hiranuma et al., 2015). Alternatively, the ice nucleation efficiency can be empirically parameterized using laboratory data, although with similar caveats (Knopf and Alpert, 2013; Murray et al., 2012). 
Molecular Dynamics (MD) simulations and direct kinetic methods have been used to study ice nucleation (e.g., Taylor and Hale, 1993; Matsumoto et al., 2002; Lupi et al., 2014; Espinosa et al., 2014). However, the classical nucleation theory (CNT) is nearly the only theoretical approach employed to describe immersion freezing in cloud models (e.g., Khvorostyanov and Curry, 2004; Barahona and Nenes, 2008, 2009; Hoose et al., 2010). According to CNT, nucleation is initiated by the growth of a cap-shaped ice germ on the surface of the immersed particle (Pruppacher and Klett, 1997; Kashchiev, 2000). The geometry of the ice germ is defined by a force balance at the particle-ice-liquid interface and is characterized by the contact angle, $\theta$. In this sense, the ice germ is assumed to "wet" the immersed particle in the same way a liquid droplet wets a solid surface (De Gennes, 1985). Low values of $\theta$ indicate a high affinity of the particle for ice and a low energy of formation of the ice germ.

Direct application of CNT in immersion freezing is thwarted by uncertainty in fundamental parameters of the theory, i.e., the ice-liquid interfacial energy, $\sigma_{\text {iw }}$, and the activation energy. Moreover, using a single $\theta$ to describe the nucleation efficiency of dust and other materials typically leads to a large discrepancy between CNT predictions and experimental measurements (e.g., Zobrist et al., 2007; Alpert et al., 2011; Broadley et al., 2012; Rigg et al., 2013). MD simulations show that an ice germ formed near a surface tends to have a complex geometry instead of the cap-shaped assumption of CNT (e.g., Lupi et al., 2014; Cox et al., 2015; Fitzner et al., 2015). Within a liquid the ice germ may not "wet" the particle but may rather exert stress on the substrate (Cahn, 1980; Rusanov, 2005), and it is not clear that this can be described as a simple function of $\theta$ (Cahn, 1980). It has also been shown that $\sigma_{\text {iw }}$ obtained by fitting CNT to measured nucleation rates tend to be biased to account for the mixing effects neglected in common formulations of the theory (Barahona, 2014).

More fundamentally, CNT neglects important interactions near the immersed particle that may influence the nucleation rate. It is assumed that ice nucleation solely depends on the local geometry of the absorbed molecules on the immersed particle (Kashchiev, 2000). This implies that the particle influences the formation of the ice germ but does not influence its adjacent water. The viscosity and density of water in the vicinity of the particle and in contact with the ice germ are assumed similar to those in the bulk (Kashchiev, 2000; Pruppacher and Klett, 1997). This is at odds with evidence of a strong effect of immersed particles on the vicinal water (Drost-Hansen, 1969; Michot et al., 2002). In fact, such an effect may be responsible for the enhancement of ice nucleation near immersed solids (Anderson, 1967).

\subsection{Evidence for the formation of ordered structures near the liquid-particle interface}

It has been known for some time that water near interfaces displays physicochemical properties different from those of the bulk (e.g., Drost-Hansen, 1969; Michot et al., 2002; Bellissent-Funel, 2002). By examining a wealth of reported experimental observations, Drost-Hansen (1969) concluded that vicinal water (i.e., the water immediately adjacent to the particle) may exist in an ordered state near the solidliquid interface that may propagate over considerable distance, of the order of hundreds to thousands of molecular diameters. More recent experiments showing that hydrophilic surfaces have a long-range impact further support this conclusion (e.g., Zheng et al., 2006). The interaction between the particle and the vicinal water becomes more significant as the temperature decreases and the viscosity increases (Wolfe et al., 2002). Recent studies have shown the presence of ordered water near the interface of biological (Cooke and Kuntz, 1974; Snyder et al., 2014), metallic (Michot et al., 2002) and clay (Yu et al., 2001; Rinnert et al., 2005) particles, a notion that is also supported by molecular dynamics simulations (Lupi et al., 2014; Cox et al., 2015). In a groundbreaking work, Anderson (1967) found strong evidence of ice formation several molecular diameters away from the clay-water interface. The author concluded that ice formation does not require an ice germ attached to the substrate, but rather the nascent ice germ is stabilized by ordering in the interfacial zone. To date no quantitative theory has been developed exploiting such a view of ice nucleation.

The description of the properties of the vicinal water is still under investigation. In the supercooled region the presence of structured low-density water near solid surfaces (termed "ice-like") has been reported for different materials (e.g., Etzler, 1983; Yu et al., 2001; Michaelides and Morgenstern, 2007; Feibelman, 2010; Snyder et al., 2014). In this region Etzler (1983) parameterized the density and enthalpy of vicinal water as a mixture of ice-like and bulk-like water. Additional experimental observations also show the modification of the mobility of water near interfaces and a higher viscosity than the bulk (Warne et al., 2000; Yu et al., 2001; Wolfe et al., 2002; Wang et al., 2006). In some cases, clays and biological systems exhibit a viscous layer of water at the particle-liquid interface that remains liquid even if the bulk has already frozen (Drost-Hansen, 1969). These effects are typically characterized as non-equilibrium, since they affect the flux of molecules to the nascent ice germ rather than the thermodynamics of ice nucleation. Li et al. (2014) found experimentally that the viscosity of interfacial water regulates the ice nucleation activity, giving support to the idea that the work of nucleation and the enhancement of the viscosity in the interfacial region are tightly linked. In fact, increased viscosity may be a necessary condition for ice nucleation, since structural ordering is not possible in a fluid with low viscosity (Anderson, 1967). 
These considerations are largely missing in the theoretical description of ice nucleation. There is currently no theory that can account for the thermodynamic and kinetic effects of an immersed particle on the surrounding water, hence on ice nucleation. Such a task is undertaken in this work. Section 2 presents the theoretical description of a new approach, accounting for the thermodynamics of vicinal water (Sect. 2.3) and its relation to the work of nucleation (Sect. 2.4) and the nucleation rate (Sects. 2.5 and 2.6). These new relations are analyzed and applied to specific cases of atmospheric relevance in Sect. 3.

\section{Theoretical development}

The new approach is developed within the scope of the kinetic treatment of nucleation, when cluster formation is the limiting step to ice formation (Pruppacher and Klett, 1997; Kashchiev, 2000). The central result of this theory is the following well-known general expression for the nucleation rate in steady state (e.g., Kashchiev, 2000, cf. Eq. 13.33):

$J=Z f^{*} C^{*}$,

where $Z$ is the Zeldovich factor, $f^{*}$ is the attachment frequency (also called the impingement factor), and $C^{*}$ is the concentration of supercritical clusters. $Z$ corrects for the detachment of monomers from the cluster during nucleation. It can also be interpreted as the probability that a molecule reaches the ice germ following a thermally activated "random walk". Generally,

$Z=\left[-\frac{\left(\frac{\partial^{2} \Delta G}{\partial n^{2}}\right)_{n=n^{*}}}{2 \pi k_{B} T}\right]^{1 / 2}$,

where $\Delta G$ is the work of cluster formation and $n^{*}$ is the number of water molecules in the ice germ. If the molecular cluster size distribution can be assumed to be near equilibrium, which is the case for immersion freezing, then

$C^{*}=C_{0} \exp \left(-\frac{\Delta G^{*}}{k_{B} T}\right)$,

where $\Delta G^{*}$ is the work of critical germ formation and $C_{0}$ is the monomer concentration adjacent to the surface of the growing ice germ, implying that interface transfer is the dominant mechanism of cluster growth.

These expressions can be applied directly to model ice nucleation as follows. For homogeneous ice nucleation, $\Delta G^{*}=$ $\Delta G_{\text {hom }}$ and $f^{*}=f_{\text {hom }}^{*}$, with $C_{0}=v_{\mathrm{w}}^{-1} v_{\mathrm{w}}$ being the molecular volume of water (Pruppacher and Klett, 1997):

$J_{\text {hom }}=\frac{Z f_{\text {hom }}^{*}}{v_{\mathrm{w}}} \exp \left(-\frac{\Delta G_{\mathrm{hom}}}{k_{B} T}\right)$.
For heterogeneous ice nucleation, $\Delta G^{*}=\Delta G_{\text {het }}$ and $f^{*}=$ $f_{\text {het }}^{*}$, with $C_{0}=a_{0}^{-1}$ being $a_{0}$, the average cross-sectional area of a water molecule, i.e.,

$J_{\text {het }}=\frac{Z f_{\text {het }}^{*}}{a_{0}} \exp \left(-\frac{\Delta G_{\text {het }}}{k_{B} T}\right)$.

Using $C_{0}=a_{0}^{-1}$ is advantageous, because $J_{\text {het }}$ is typically normalized to the particle surface area (Murray et al., 2012; Hoose and Möhler, 2012). It, however, involves the assumption that the density of water does not vary within the droplet, remaining constant even at the particle-water interface. In other words, anywhere within the liquid, the per-area molecular density should be the same. This assumption, however, does not lead to significant error, since the effect of the particle on the water density is small (e.g., Etzler, 1983), and $J_{\text {het }}$ is linearly related to $C_{0}$.

Equation (5) provides the basis for this work. It shows that, to predict the effect of the immersed particle on ice formation, it is necessary to understand how the presence of the particle affects $\Delta G_{\text {het }}$ and $f_{\text {het }}^{*}$. To accomplish this Sect. 2.1 provides an overview of the main assumptions of CNT, which are then contrasted with the negentropic nucleation framework (NNF) in Sect. 2.2. The latter is used in Sects. 2.3 and 2.4 to analyze the thermodynamic aspects of immersion ice nucleation and to formulate a new expression for $\Delta G_{\text {het }}$. Section 2.5 develops an expression for $f_{\text {het }}^{*}$ accounting for the effect of the particle on the mobility of water molecules. In Sect. 2.6 a new expression for the nucleation rate is formulated.

\subsection{Classical nucleation theory}

Since CNT is the most widely used theoretical approach in atmospheric models we start by highlighting its main characteristic. Common CNT expressions use several assumptions to simplify the description of the interaction between water and the immersed particle (e.g., Khvorostyanov and Curry, 2004; Zobrist et al., 2007; Hoose et al., 2010). Typically the particle is assumed to have a negligible effect on the mobility and the thermodynamics of the vicinal water, i.e., $f_{\text {het }}^{*} \approx f_{\text {hom }}^{*}$. The latter is calculated assuming that the formation of clusters within the liquid phase mimics a first-order reaction in an ideal gas where every molecule that randomly jumps into the ice-liquid interface is incorporated within the ice lattice. Thus $f_{\mathrm{hom}}^{*}$ is the product of the frequency factor (derived from transition state theory) and the monomer concentration at the ice-liquid interface. This leads to (Pruppacher and Klett, 1997; Kashchiev, 2000)

$f_{\text {het,CNT }}^{*}=f_{\text {hom,CNT }}^{*}=\frac{\Omega d_{0}}{v_{\mathrm{w}}} \frac{k_{B} T}{h} \exp \left[-\frac{\Delta G_{\text {act }}}{k_{B} T}\right]$,

where $\Delta G_{\text {act }}$ is the activation energy, i.e., the energy required for a water molecule to leave its equilibrium position in the bulk towards the vicinity of the ice germ (Pruppacher and 
Klett, 1997; Zobrist et al., 2007), $h$ is Plank's constant, $\Omega$ the surface area of the ice germ and $d_{0}$ is the molecular diameter of water.

The work of ice nucleation results from the assumption that the ice germ has a hemispherical shape. Other assumptions include no surface stress (Cahn, 1980) and negligible mixing effects during germ formation (Barahona, 2014). These considerations lead to the expression (Turnbull and Fisher, 1949)

$$
\Delta G_{\mathrm{het}, \mathrm{CNT}}=g(\theta) \Delta G_{\mathrm{hom}, \mathrm{CNT}},
$$

where $\Delta G_{\text {hom,CNT }}$ is the homogeneous work of nucleation, given by

$\Delta G_{\mathrm{hom}, \mathrm{CNT}}=\frac{16 \pi \sigma_{\mathrm{iw}}^{3} v_{\mathrm{w}}^{2}}{3\left(k_{B} T \ln S_{\mathrm{i}}\right)^{2}}$,

where $\sigma_{\text {iw }}$ is the ice-water interfacial energy and $S_{\mathrm{i}}$ is the saturation ratio with respect to ice. The effect of the immersed particle on $J_{\text {het,CNT }}$ depends on the adsorption of water molecules on individual sites, and is characterized by the contact angle, $\theta$, in the form

$g(\theta)=\frac{1}{4}(2+\cos \theta)(1-\cos \theta)^{2}$.

Equation (9) can be extended to account for line tension, curvature and misfit effects (e.g., Khvorostyanov and Curry, 2004), which, however, requires the usage of additional unconstrained parameters. Introducing Eqs. (6) and (7) into Eq. (5) we obtain the known expression,

$$
J_{\mathrm{het}, \mathrm{CNT}}=\frac{Z \Omega d_{0}}{a_{0} v_{\mathrm{w}}} \frac{k_{B} T}{h} \exp \left[-\frac{\Delta G_{\mathrm{act}}+g(\theta) \Delta G_{\mathrm{hom}, \mathrm{CNT}}}{k_{B} T}\right],
$$

where $\Omega=4 \pi r_{\mathrm{g}}^{2}$, and $r_{\mathrm{g}}=\left(\frac{3 n^{*} v_{\mathrm{w}}}{4 \pi}\right)^{1 / 3}$ is the radius of the ice germ. Other symbols are defined in Table 1 .

Due in part to the assumption of a negligible effect of the particle on the adjacent water the CNT framework does not provide a way to link the properties of the vicinal water to the nucleation rate. Another caveat of CNT is that fundamental parameters like $\Delta G_{\text {act }}, \sigma_{\text {iw }}$ and $\theta$ do not have a clear definition outside of the context of the theory. For example, $\Delta G_{\text {act }}$ is typically assumed the same as in bulk water, representing a barrier to bulk diffusion instead of interfacial transfer (Kashchiev, 2000; Barahona, 2015). Similarly $\sigma_{\text {iw }}$ is not well defined for a diffuse interface, and it is difficult to measure away from equilibrium. Moreover, $\theta$ relies on a droplet-like picture of the nascent ice germ, which may not be appropriate for a germ forming within the dense liquid phase (Brukhno et al., 2008). Most studies thus treat $\Delta G_{\text {act }}, \sigma_{\text {iw }}$ and $\theta$ as empirical parameters, fitted to match measured nucleation rates. Many times this results in complex functional forms of $T$ and $S_{\mathrm{i}}$ that may not be easily expanded to account for the modified properties of water near the immersed particle.

\subsection{Negentropic nucleation framework}

Some of the caveats of CNT are addressed in the negentropic nucleation framework (NNF; Barahona, 2014, 2015). In NNF simple thermodynamic arguments are used to approximate $\Delta G_{\text {hom }}$ and $f_{\text {hom }}$ in terms of water properties that could, in principle, be independently estimated. This obviates the need for parameters that should be fitted to measured nucleation rates. At the same time, NNF is a relatively simple framework that can be easily implemented in large-scale atmospheric models and that has been shown to reproduce homogeneous freezing temperatures down to $180 \mathrm{~K}$ (Barahona, 2015; O and Wood, 2016). This section presents the main results of NNF for homogeneous ice nucleation.

In NNF the energy of formation of the interface, $\Phi_{\mathrm{S}}$, is an explicit function of the water activity and temperature in the form

$\Phi_{\mathrm{s}}=\Gamma_{\mathrm{w}} s\left(\Delta h_{\mathrm{f}}-\Gamma_{\mathrm{w}} k_{B} T \ln a_{\mathrm{w}}\right)$,

where the constants $\Gamma_{\mathrm{w}}=1.46$ and $s=1.105 \mathrm{molec}^{1 / 3} \mathrm{de}$ fine the coverage of the ice-water interface and the lattice geometry of the ice germ, respectively, and $\Delta h_{\mathrm{f}}$ is the latent heat of fusion of water. Other symbols are defined in Appendix A. Equation (11) results from accounting for the finite character of the ice-liquid interface and from the assumption that, in joining the ice lattice, the water molecules lose most of their entropy (Barahona, 2014). The driving force for ice nucleation, $\Delta \mu_{\mathrm{i}}$, is given by

$\Delta \mu_{\mathrm{i}}=k_{B} T \ln \left(\frac{a_{\mathrm{w}}^{2}}{a_{\mathrm{w}, \mathrm{eq}}}\right)$,

where $a_{\mathrm{w}, \text { eq }}$ is the equilibrium water activity. Equation (12) accounts for the work of "unmixing" affecting the bulk of the liquid when the ice germ is formed, which is proportional to $\ln \left(a_{\mathrm{w}}\right)$ (Black, 2007). Using Eqs. (11) and (12), the critical germ size and the work of nucleation are obtained from the condition of mechanical equilibrium of the ice germ (Barahona, 2014), resulting in

$n_{\text {hom }}=\left(\frac{2 \Phi_{\mathrm{s}}}{3 \Delta \mu_{\mathrm{i}}}\right)^{3}$,

and

$\Delta G_{\mathrm{hom}, \mathrm{NNF}}=\frac{4}{27} \frac{\Phi_{\mathrm{s}}^{3}}{\Delta \mu_{i}^{2}}=\frac{1}{2} n_{\mathrm{hom}} \Delta \mu_{\mathrm{i}}$.

In more recent work the kinetics of homogeneous ice nucleation have been re-examined in NNF to account for molecular rearrangement during the transfer of water molecules across the ice-liquid interface (Barahona, 2015). Within this approach $f_{\text {hom }}^{*}$ is determined by the diffusion coefficient for interfacial transfer, $D$, in the form (Kashchiev, 2000; Barahona, 2015)

$f^{*}=\frac{D \Omega}{v_{\mathrm{w}} d_{0}}$, 
where $\Omega$ is the surface area of the ice germ. $D$ represents contributions from purely diffusive process and from structural transformations required to incorporate water molecules into the ice germ. The latter originates because neighboring molecules need to be rearranged to accommodate new ones into the ice lattice, generating entropy and dissipating work. Using considerations from non-equilibrium thermodynamics $D$ can be written in the form (Barahona, 2015)

$D=D_{\infty}\left[1+\exp \left(\frac{W_{\mathrm{d}}}{k_{B} T}\right)\right]^{-1}$,

where $D_{\infty}$ is the bulk self-diffusion coefficient of water, and $W_{\mathrm{d}}$ is the average dissipated work during interface transfer. The latter is proportional to the excess free energy of solidification of water, i.e., $W_{\mathrm{d}}=-n_{\mathrm{t}} \Delta \mu_{\mathrm{s}}$, with $n_{\mathrm{t}}=16$, the number of possible trajectories in which individual water molecules can make four-bonded water. Equation (16) shows explicitly that bulk diffusion (i.e., $D_{\infty}$ ) as well as structural rearrangement are required for ice germ growth. Introducing Eq. (16) into Eq. (15) we obtain

$f_{\text {hom }}^{*}=\frac{D_{\infty} \Omega}{v_{\mathrm{w}} d_{0}}\left[1+\exp \left(-n_{\mathrm{t}} \Delta \mu_{\mathrm{s}}\right)\right]^{-1}$.

Application of Eq. (17) to homogeneous ice nucleation shows agreement of $J_{\text {hom }}$ with experimental data at very low $T$, where kinetic processes dominate the formation ice (Barahona, 2015).

NNF provides explicit dependencies of $D$ and $\Phi_{\mathrm{s}}$ on thermodynamic properties without depending on nucleation rate measurements. Thus it provides a suitable basis to study the thermodynamics and kinetics of ice formation in the vicinity of immersed particles. Doing so first requires building a model to describe the thermodynamics of the vicinal water.

\subsection{Thermodynamics of the liquid-particle interface}

The discussion presented in Sect. 1.1 suggests that the immersed particle enhances order near the particle-liquid interface, lowering the energy required to nucleate ice. The vicinal layer is thus described as a solution of hypothetical icelike (IL) and liquid-like (LL) regions, with Gibbs free energy, given by

$\mu_{\mathrm{vc}}=(1-\zeta) \hat{\mu}_{\mathrm{LL}}+\zeta \hat{\mu}_{\mathrm{IL}}$,

where $\hat{\mu}_{\mathrm{LL}}$ and $\hat{\mu}_{\mathrm{LL}}$ are the chemical potentials of the LL and IL species within the solution, respectively, and $\zeta$ is the fraction of IL regions in the layer. Increased order is represented by a higher fraction of IL regions, hence higher $\zeta$. Equation (18) can also be written in terms of the chemical potentials of the "pure" LL and IL species, $\mu_{\mathrm{LL}}$ and $\mu_{\mathrm{IL}}$, respectively, in the form

$\mu_{\mathrm{vc}}=(1-\zeta) \mu_{\mathrm{LL}}+\zeta \mu_{\mathrm{IL}}+\Delta G_{\mathrm{mix}}$, where $\Delta G_{\text {mix }}=\left(\hat{\mu}_{\mathrm{IL}}-\mu_{\mathrm{IL}}\right) \zeta+(1-\zeta)\left(\hat{\mu}_{\mathrm{LL}}-\mu_{\mathrm{LL}}\right)$ is the Gibbs energy of mixing. For a mechanical mixture of pure LL and IL species, $\Delta G_{\text {mix }}=0$, whereas for an ideal solution, $\Delta G_{\text {mix }}$ is determined by the ideal entropy of mixing (Prausnitz et al., 1998). Reorganizing Eq. (19) we obtain,

$\mu_{\mathrm{vc}}=\mu_{\mathrm{LL}}+\zeta \Delta \mu_{\mathrm{il}}+\Delta G_{\mathrm{mix}}$,

where $\Delta \mu_{\mathrm{il}}=\mu_{\mathrm{IL}}-\mu_{\mathrm{LL}} \cdot \Delta \mu_{\mathrm{il}}$ can be approximated using the equilibrium between bulk liquid and ice as a reference state (Kashchiev, 2000), making

$\mu_{\mathrm{IL}}=\mu_{\mathrm{eq}}+k_{B} T \ln \left(a_{\mathrm{IL}}\right)$,

and

$\mu_{\mathrm{LL}}=\mu_{\mathrm{eq}}+k_{B} T \ln \left(\frac{a_{\mathrm{w}, \mathrm{eff}}}{a_{\mathrm{w}, \mathrm{eq}}}\right)$,

where $a_{\mathrm{w}, \text { eff }}$ is termed the "effective water activity" and is the value of $a_{\mathrm{w}}$ associated with the LL regions in the vicinal water, and $a_{\mathrm{IL}}$ is the water activity in the IL regions. Assuming that, similarly to bulk ice, the solute does not significantly partition to the IL phase, then $a_{\mathrm{IL}} \approx 1$. With this, and by combining Eq. (21) and Eq. (22) and rearranging, we obtain

$\Delta \mu_{\mathrm{il}}=-k_{B} T \ln \left(\frac{a_{\mathrm{w}, \mathrm{eff}}}{a_{\mathrm{w}, \mathrm{eq}}}\right)$.

The central assumption behind Eq. (23) is that $a_{\mathrm{w}, \text { eq }}$ corresponds to the equilibrium water activity between liquid and ice, or in other words, that near equilibrium $\Delta \mu_{\mathrm{il}} \approx \Delta \mu_{\mathrm{s}}$. In reality $\Delta \mu_{\mathrm{S}}$ corresponds to actual liquid and ice, instead of the hypothetical LL and IL substances. This difference can be accounted for by selecting a proper functional form for $\Delta G_{\text {mix }}$, for which several empirical and semi-empirical interaction models, with varying degrees of complexity, exist (Prausnitz et al., 1998). In this work it is assumed that the vicinal water can be described as a regular solution. This is the simplest model that accounts for the interaction between solvent and solute during mixing and that is flexible enough to include corrections for the difference between $\Delta \mu_{\mathrm{s}}$ and $\Delta \mu_{\mathrm{il}}$. Using this model Holten et al. (2013) were able to approximate the chemical potential of supercooled water. The authors also showed that taking into account clustering of water molecules led to a better agreement of the estimated water properties with MD simulations and experimental results.

According to the regular solution model, modified by clustering (Holten et al., 2013, cf. Eq. 16),

$$
\begin{aligned}
\Delta G_{\operatorname{mix}} & =\frac{k_{B} T}{N}[\zeta \ln (\zeta)+(1-\zeta) \ln (1-\zeta)] \\
& +A_{\mathrm{w}} \zeta(1-\zeta) .
\end{aligned}
$$

The first term on the right-hand side corresponds to the usual definition of the ideal entropy of mixing, i.e., random ideal 
mixing and weak interaction between IL and LL regions, modified to account for clustering in groups of $N$ molecules. $N=6$ corresponds to clustering in hexamers and is near the optimum fit between MD simulations and the solution model (Holten et al., 2013). It must be noted that Holten et al. (2013) recommended an alternative model termed "athermal solution", where nonideality is ascribed to entropy changes upon mixing. In vicinal water some evidence points to nonideality originating from enthalpy changes near the particle (Etzler, 1983); hence a regular solution is more appropriate in this case. For $N=6$ the difference between the two models is negligible (Holten et al., 2013).

The second term on the right-hand side of Eq. (24) is an empirical functional form used to approximate the enthalpy of mixing, selected so that $\Delta G_{\text {mix }}=0$ for both $\zeta=0$ and $\zeta=1 . A_{\mathrm{w}}$ is a phenomenological interaction parameter, here assumed to implicitly correct the approximation $\Delta \mu_{\mathrm{il}} \approx \Delta \mu_{\mathrm{s}}$. Typically $A_{\mathrm{w}}$ must be fitted to experimental observations. In this work $A_{\mathrm{w}}$ is calculated using an alternative approach, as follows.

An important aspect of the regular solution model is that it predicts that $\mu_{\mathrm{vc}}$ has a critical temperature, $T_{\mathrm{c}}$, defined by the conditions,

$$
\frac{\partial^{2} \mu_{\mathrm{vc}}}{\partial \zeta^{2}}=0, \frac{\partial^{3} \mu_{\mathrm{vc}}}{\partial \zeta^{3}}=0
$$

These conditions originate because $\frac{\partial^{2} \mu_{\mathrm{vc}}}{\partial \zeta^{2}}=0$ represents a stability limit for the vicinal water. A solution would split into two phases, if doing so lowers its Gibbs free energy (Prausnitz et al., 1998, cf. Sect. 6.12). For a metastable solution $\mu_{\mathrm{vc}}$ must be minimal, hence $\frac{\partial \mu_{\mathrm{vc}}}{\partial \zeta}=0$. The condition $\frac{\partial^{2} \mu_{\mathrm{vc}}}{\partial \zeta^{2}}<0$ indicates that any increase in $\zeta$ increases $\mu_{\mathrm{vc}}$ (i.e., the curve $\mu_{\mathrm{vc}}$ vs. $\zeta$ becomes concave downward), such that it is thermodynamically more favorable for the solution to split into distinct phases than to increase its concentration. The last condition, $\frac{\partial^{3} \mu_{\mathrm{Vc}}}{\partial \zeta^{3}}=0$, indicates that the metastable region reduces to a single point. Using Eq. (20) into Eq. (25) we obtain,

$$
\frac{\partial^{2} \mu_{\mathrm{vc}}}{\partial \zeta^{2}}=\frac{k_{B} T}{N}\left(\frac{1}{\zeta(1-\zeta)}\right)-2 A_{\mathrm{w}}=0
$$

and

$$
\frac{\partial^{3} \mu_{\mathrm{vc}}}{\partial \zeta^{3}}=\frac{k_{B} T}{N}\left(\frac{\zeta^{2}-(1-\zeta)^{2}}{\zeta^{2}(1-\zeta)^{2}}\right)=0 .
$$

The last expression is only valid for $\zeta=0.5$, indicating that a single critical temperature exists for a regular solution. Using this in Eq. (26) and solving for $A_{\mathrm{w}}$ gives, for $T=T_{\mathrm{c}}$,

$A_{\mathrm{w}}=\frac{2 k_{B} T_{\mathrm{c}}}{N}$.

Physically, $T_{\mathrm{c}}$ represents the stability limit of the vicinal water, at which it spontaneously separates into IL and LL re- gions. For $T<T_{\mathrm{c}}$ the chemical potential of an equimolar solution of IL and LL would be larger than that of a simple mechanical mixture of the two species. Thus it is thermodynamically more favorable for the solution to split into its individual components, i.e., ice and liquid, leading to a stability limit of the system. Equation (28) thus provides an opportunity to theoretically determine $A_{\mathrm{w}}$, since $T_{\mathrm{c}}$ should also correspond to a negligible work of nucleation. This further explained in Sect. 2.4.2.

Introducing Eqs. (23), (24) and (28) in Eq. (20), we obtain

$$
\begin{aligned}
\mu_{\mathrm{vc}} & =\mu_{\mathrm{LL}}-\zeta k_{B} T \ln \left(\frac{a_{\mathrm{w}, \mathrm{eff}}}{a_{\mathrm{w}, \mathrm{eq}}}\right) \\
& +\frac{k_{B} T}{N}[\zeta \ln (\zeta)+(1-\zeta) \ln (1-\zeta)]+\frac{2 k_{B} T_{\mathrm{c}}}{N} \zeta(1-\zeta) .
\end{aligned}
$$

By defining $\Lambda_{\text {mix }}$ in the form,

$\Lambda_{\text {mix }}=\frac{1}{N}[\zeta \ln (\zeta)+(1-\zeta) \ln (1-\zeta)]+\frac{2}{N} \frac{T_{\mathrm{c}}}{T} \zeta(1-\zeta)$,

Equation (29) can be written in the form

$\mu_{\mathrm{vc}}=\mu_{\mathrm{LL}}-\zeta k_{B} T \ln \left(\frac{a_{\mathrm{w}, \mathrm{eff}}}{a_{\mathrm{w}, \mathrm{eq}}}\right)+k_{B} T \Lambda_{\mathrm{mix}}$.

Equation (31) is the equation of state of the vicinal water. It describes the properties of the vicinal water in terms of the material-specific parameter $\zeta$ and the interaction parameters $N$ and $T_{\mathrm{c}}$. MD simulations indicate that $N \sim 6$ (Bullock and Molinero, 2013; Holten et al., 2013). $T_{\mathrm{c}}$ is thus the only remaining unknown in Eq. (31) and is calculated in Sect. 2.4.2.

\subsection{Work of germ formation}

The equation of state of vicinal water can be used to link $\Delta G_{\text {hom }}$ and $\Delta G_{\text {het }}$ as follows. In immersion freezing the particle remains within the droplet long enough that equilibrium is established. This condition is mathematically expressed by the equality, $\mu_{\mathrm{vc}}=\mu_{\mathrm{w}}$, where $\mu_{\mathrm{w}}$ is the chemical potential of water in the bulk of the liquid, i.e., away from the particle. Using Eq. (31) this implies that

$\mu_{\mathrm{w}}=\mu_{\mathrm{LL}}-\zeta k_{B} T \ln \left(\frac{a_{\mathrm{w}, \mathrm{eff}}}{a_{\mathrm{w}, \mathrm{eq}}}\right)+k_{B} T \Lambda_{\mathrm{mix}}$.

This expression indicates that the effect of the particle on its vicinal water can be understood as an enhancement of the chemical potential of the LL regions, a consequence of the tendency of the particle to lower $\mu_{\mathrm{vc}}$. Since $\Delta \mu_{\mathrm{il}}<0, \mu_{\mathrm{LL}}$ must increase to maintain equilibrium. Using the equilibrium between bulk liquid and ice as reference state so that $\mu_{\mathrm{w}}=$ $\mu_{\text {eq }}+k_{B} \ln \left(\frac{a_{\mathrm{w}}}{a_{\mathrm{w}, \mathrm{eq}}}\right)$, we obtain the following after simplifying:

$\ln \left(a_{\mathrm{w}}\right)=\ln \left(a_{\mathrm{w}, \mathrm{eff}}\right)-\zeta \ln \left(\frac{a_{\mathrm{w}, \mathrm{eff}}}{a_{\mathrm{w}, \mathrm{eq}}}\right)+\Lambda_{\text {mix }}$. 
Or, equivalently,

$a_{\mathrm{w}}=a_{\mathrm{w}, \text { eff }}\left(\frac{a_{\mathrm{w}, \mathrm{eq}}}{a_{\mathrm{w}, \mathrm{eff}}}\right)^{\zeta} \exp \left(\Lambda_{\mathrm{mix}}\right)$.

Equation (34) suggests that, thermodynamically, immersion freezing can be described as homogeneous ice nucleation occurring at an enhanced water activity. This is because it is possible to create a path including homogeneous ice nucleation with the same change in Gibbs free energy as for heterogeneous freezing. Figure 1 shows that for a particledroplet system in equilibrium, $a_{\mathrm{w} \text {,eff }}$ satisfies the condition

$\Delta G_{\text {het }}\left(a_{\mathrm{w}}\right)=\Delta G_{\mathrm{hom}}\left(a_{\mathrm{w}, \mathrm{eff}}\right)$.

Equation (35) represents a thermodynamic relation between

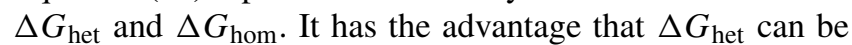
obtained without invoking assumptions on the mechanistic details of the interaction between the particle and the ice germ, which are parameterized by $\zeta$. Since $a_{\mathrm{w}}$ is typically the controlled variable in ice nucleation, $a_{\mathrm{w}, \text { eff }}$ can be readily obtained by solving Eq. (34),

$a_{\mathrm{w}, \mathrm{eff}}=\left(\frac{a_{\mathrm{w}}}{a_{\mathrm{w}, \mathrm{eq}}^{\zeta}}\right)^{\frac{1}{1-\zeta}} \exp \left(-\frac{\Lambda_{\text {mix }}}{1-\zeta}\right)$.

Although ascribing ice nucleation to the LL fraction of vicinal water agrees with the decisive role of free water in the formation of ice (Wang et al., 2016), caution must be taken in considering this to be the actual mechanism of ice nucleation, which could be quite complex. Equation (35), however, establishes a thermodynamic constrain for $\Delta G_{\text {het }}$ that should be met by any ice nucleation mechanism. It is also important to analyze the behavior of $a_{\mathrm{w} \text {,eff }}$ as $\zeta \rightarrow 1$. It can be shown that the quotient $\frac{\Lambda_{\text {mix }}}{\zeta-1}$ converges for $\zeta \rightarrow 1$ as follows. From Eq. (30) we can write

$\frac{\Lambda_{\mathrm{mix}}}{1-\zeta}=\frac{1}{N(1-\zeta)}[\zeta \ln (\zeta)+(1-\zeta) \ln (1-\zeta)]+\frac{2}{N} \frac{T_{\mathrm{c}}}{T} \zeta$.

Using $\ln (x) \rightarrow(x-1)$ for $x \rightarrow 1$, the last expression can be shown to converge to

$$
\begin{aligned}
\lim _{\zeta \rightarrow 1} \frac{\Lambda_{\text {mix }}}{1-\zeta} & =-\frac{2 \zeta}{N} \\
& +\frac{2}{N} \frac{T_{\mathrm{c}}}{T} \zeta=\frac{2}{N}\left(\frac{T_{\mathrm{c}}}{T}-1\right) .
\end{aligned}
$$

The fact that $\lim _{\zeta \rightarrow 1} \exp \left(-\frac{\Lambda_{\text {mix }}}{1-\zeta}\right) \neq 1$ for $T \neq T_{\mathrm{c}}$ stems from the simple interaction model used to define $\Delta G_{\text {mix }}$ (i.e., the regular solution approximation). $T_{\mathrm{c}}$ may depend on $\zeta$, however the regular solution approximation predicts a unique critical temperature at $\zeta=0.5$. This, however, does not lead to uncertainty in $\Delta G_{\text {hom }}$ since for $\zeta \rightarrow 1$, the first term on the right-hand side of Eq. (36) is almost singular at $a_{\mathrm{w}}=a_{\mathrm{w} \text {,eq. }}$ Thus if $\lim _{\zeta \rightarrow 1} \exp \left(-\frac{\Lambda_{\text {mix }}}{1-\zeta}\right)<1$, then $a_{\mathrm{w}}$ must be just above $a_{\mathrm{w}, \mathrm{eq}}$ to make $a_{\mathrm{w}, \mathrm{eff}}=1$. In other words, for all practical purposes, $a_{\mathrm{w}, \text { eff }} \rightarrow 1$ when the system approaches thermodynamic equilibrium.

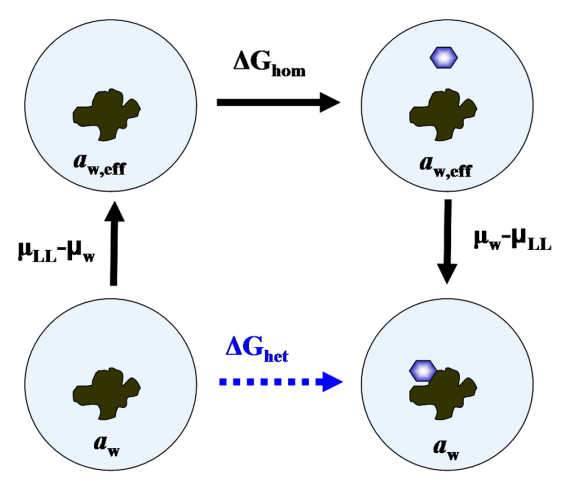

Figure 1. Diagram representing a thermodynamic path, including homogeneous ice nucleation with the same work as heterogeneous freezing.

\subsubsection{Extension of the homogeneous model to the spinodal limit}

In applying the homogeneous model to the heterogeneous problem in the form detailed in Sect. 2.4, caution must be taken in describing the limiting condition where the size of the ice germ becomes exceedingly small, i.e., $n_{\text {hom }} \rightarrow 1$, representing the vanishing of the energy barrier to ice nucleation. This is possible, since as $\zeta \rightarrow 1, a_{\mathrm{w} \text {,eff }}$ becomes large (Eq. 36), and for $\zeta=1$ it is only defined at thermodynamic equilibrium. Since for $n_{\text {hom }} \rightarrow 1$, thermodynamic potentials are not well defined, it is necessary to test the validity of $\mathrm{NNF}$ at such a limit. Moreover, in its original formulation (Sect. 2.2) NNF predicts a positive $\Delta G_{\text {hom }}$ for $n_{\text {hom }}=1$, at odds with the notion that the formation of a monomer-sized germ should carry no work.

At the limiting condition, $n_{\text {hom }}=1$, the work of nucleation is smaller than the thermal energy of the molecules and represents the onset of spontaneous phase separation (termed "spinodal decomposition") during nucleation (Vekilov, 2010). Here it is argued that being a far-fromequilibrium process, ice nucleation always carries energy dissipation. When accounted for, the apparent inconsistency in NNF at $n_{\text {hom }}=1$ vanishes, since as shown below such a condition is not accessible. This approach differs from previous treatments, where this limit is associated with a negligible driving force for nucleation (Kalikmanov and van Dongen, 1993).

To account for the finite, albeit small, amount of work dissipated from the generation of entropy during spontaneous fluctuation, a simple approach is proposed. It involves writing the work of cluster formation in the form

$\Delta G=-n \Delta \mu_{\mathrm{i}}+n^{2 / 3} \Phi_{\mathrm{s}}+W_{\mathrm{diss}}$,

where $W_{\text {diss }}$ represents work dissipation, assumed independent of the germ size, since it results from spontaneous fluctuations occurring in the liquid phase. Equation (39) is the typical expression for $\Delta G$ (Barahona, 2014, cf. Eq. 15) with 
an additional term accounting for irreversibility. The nucleation work is defined for $n=n_{\text {hom }}$ in the form

$\Delta G_{\text {hom }}=-n_{\text {hom }} \Delta \mu_{\mathrm{i}}+n_{\text {hom }}^{2 / 3} \Phi_{\mathrm{s}}+W_{\text {diss }}$,

where $n_{\text {hom }}$ is obtained from the mechanical stability condition, $\frac{\partial \Delta G}{\partial n}=0$, and is still given by Eq. (13), since $W_{\text {diss }}$ is assumed independent of $n$. $W_{\text {diss }}$ is then obtained from the conditions

$\left.\Delta G_{\mathrm{hom}}\right|_{n_{\mathrm{hom}}=1}=0,\left.\frac{\partial^{2} \Delta G_{\mathrm{hom}}}{\partial n_{\mathrm{hom}}^{2}}\right|_{n_{\mathrm{hom}}=1}=0$.

The first condition expresses the fact that the formation of a monomer-sized ice germ carries no work. The second condition establishes that $n_{\text {hom }}=1$ should correspond to a stability limit of the system where nucleation and spontaneous separation are analogous. This is referred as the spinodal point. From Eq. (40) we obtain

$$
\frac{\partial^{2} \Delta G_{\mathrm{hom}}}{\partial n_{\mathrm{hom}}^{2}}=-\frac{2}{9} n_{\mathrm{hom}}^{-4 / 3} \Phi_{\mathrm{s}}=0
$$

Since $n_{\text {hom }}$ only attains positive values, then only the trivial solution $\Phi_{\mathrm{s}}=0$ satisfies Eq. (42), i.e., the energy barrier to the formation of the ice germ vanishes at the spinodal. Using $\Phi_{\mathrm{S}}=0$ and $\left.\Delta G_{\text {hom }}\right|_{n_{\mathrm{hom}}=1}=0$, Eq. (40) can be solved for $W_{\text {diss }}$ in the form

$W_{\mathrm{diss}}=\Delta \mu_{\mathrm{i}}$.

Thus the minimum amount of work dissipated during nucleation corresponds to a fluctuation relaxing $\Delta \mu_{\mathrm{i}}$. Replacing this expression within Eq. (40), we obtain

$\Delta G_{\mathrm{hom}}=-\Delta \mu_{\mathrm{i}}\left(n_{\mathrm{hom}}-1\right)+n_{\mathrm{hom}}^{2 / 3} \Phi_{\mathrm{s}}$

Using Eq. (13) in Eq. (44) gives, after rearranging, the work of germ formation by homogeneous ice nucleation:

$\Delta G_{\mathrm{hom}}=\frac{1}{2} \Delta \mu_{\mathrm{i}}\left(n_{\mathrm{hom}}+2\right)$.

Equation (45) only differs from the NNF expression, Eq. (14), on the right-hand side, where it is implied that nucleation in a solution requires the coordination of at least two molecules, a condition that has been observed experimentally in the crystallization of proteins (Vekilov, 2010). It also suggests that dissipation effects are negligible for typical homogeneous nucleation conditions, i.e., $\Delta G_{\mathrm{hom}} \approx \Delta G_{\mathrm{hom}, \mathrm{NNF}}$, since $n_{\text {hom }} \sim 200$ (Barahona, 2014). Moreover, the fact that $\Delta G_{\text {hom }}>0$ even when $n_{\text {hom }} \rightarrow 0$ implies that ice nucleation always requires some work. Using Eq. (35) the heterogeneous work of nucleation can be readily written as

$\Delta G_{\text {het }}\left(a_{\mathrm{w}}\right)=\left[\frac{1}{2} \Delta \mu_{\mathrm{i}}\left(n_{\mathrm{hom}}+2\right)\right]_{a_{\mathrm{w}, \mathrm{eff}}}$.

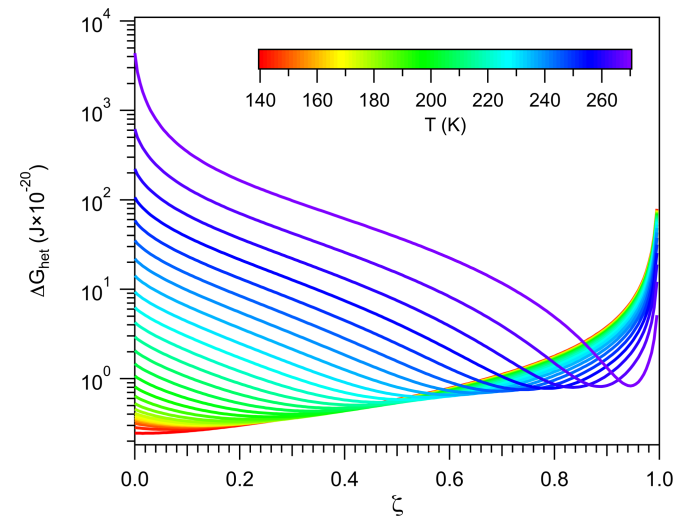

Figure 2. Work of heterogeneous ice nucleation. Color indicates different temperatures.

Equation (46) also suggests an operational definition for the critical ice germ in immersion freezing in the form

$n_{\text {het }}=\left(n_{\text {hom }}+2\right)_{a_{\mathrm{w}, \text { eff }}}$.

The results of Eqs. (45) and (46) require further explanation, since in principle, an ice germ with only two molecules does not exist. Thus Eq. (45) must be interpreted in a different way. As $\zeta \rightarrow 1$, or in deeply supercooled conditions, the fraction of ice-like regions in the vicinal water becomes large. Under such a scenario the reorientation of only two molecules may be enough to initiate ice nucleation. In other words, beyond the spinodal point ice nucleation is controlled by molecular motion within already formed ice-like regions. For homogeneous ice nucleation this would require extreme supercooling ( $T \sim 140 \mathrm{~K}$, Fig. 2). In immersion ice nucleation it may occur at higher $T$, since the formation of a high fraction of ice-like regions in the vicinal water is facilitated by efficient INPs. This is further explored in Sect. 3.

NNF carries the assumption that thermodynamic potentials can be defined for the ice germ. In other words $n_{\text {hom }}$ should be large enough that it represents a statistical ensemble of molecules. Of course this is not the case for $n_{\text {hom }}=1$, and it may cast doubt on the application of Eq. (39) to such limits. This possibility is, however, mitigated in two ways. Unlike CNT, which is based on the interfacial tension, the NNF framework is robust for small germs. Size effects impact $\Delta G$ mostly through $\Phi_{\mathrm{s}}$, since $\Delta \mu_{\mathrm{i}}$ does not change substantially with the size of the system. In NNF the product $\Gamma_{\mathrm{w}} s \Delta h_{\mathrm{f}}$ in Eq. (11) remains constant, and $\Phi_{\mathrm{s}}$ is relatively insensitive to $n$. This is because $\Delta h_{\mathrm{f}}$ decreases with $n$ as the total cohesive energy of the germ is inversely proportional to the number of molecules within the ice-liquid interfacial layer (Zhang et al., 1999; Johnston and Molinero, 2012). At the same time, the product $\Gamma_{\mathrm{w}} s$, i.e., the ratio of the number of surface to interior molecules in the germ (Barahona, 2014; Spaepen, 1975), should increase for small ice germs, offsetting the decrease in $\Delta h_{\mathrm{f}}$. Such behavior is supported 
by MD simulations (Johnston and Molinero, 2012). Equation (11) thus remains valid for small germs. A second mitigating factor is discussed in Sect. 3.1, where it is shown that conditions leading to $n_{\text {het }} \rightarrow 1$ are rare in the atmosphere, and $J_{\text {het }}$ is largely independent of $n_{\text {het }}$ for very small germs.

\subsubsection{Critical temperature}

To complete the thermodynamic description of ice nucleation near the particle-liquid interface it is necessary to specify the critical separation temperature defined in Eq. (28). The criterion used to find $T_{\mathrm{c}}$ is that the reversible work of nucleation, that is, without accounting for the dissipation term, becomes negligible. $W_{\text {diss }}$ is not included, since the definition of $\Delta G_{\text {mix }}$ (Eq. 24), does not account for such effects.

An analysis of Eqs. (44) and (46) reveals that $\Delta G_{\text {hom }}$ (hence $\Delta G_{\text {het }}$ ) is at a minimum when the reversible work becomes negligible. As $T$ decreases, $\Delta \mu_{\mathrm{i}}$ increases, decreasing $n_{\text {hom }}$ and $\Delta G_{\text {hom }}$. However, as $n_{\text {hom }} \rightarrow 0$, the tendency is reversed, since $\Delta G_{\text {hom }} \sim W_{\text {diss }}$. In this regime dissipative effects dominate, and $\Delta G_{\text {hom }}$ and $\Delta G_{\text {het }}$ become proportional to $\Delta \mu_{\mathrm{i}}$ (Fig. 2). Thus the minimum in $\Delta G_{\text {het }}$ signals $n_{\text {hom }} \rightarrow 0$ and $n_{\text {het }} \rightarrow 2$. If no dissipation or kinetic effects were present (for example at low supercooling), then phase separation would ensue, since the work of nucleation would be smaller than the thermal energy of the water molecules. This limit should also correspond to the stability limit of the vicinal water where IL and LL species separate spontaneously; hence it can be used to find $T_{\mathrm{c}}$. It must be noted that this criterion does not mean that $\Delta G_{\text {het }}=0$ at $T=T_{\mathrm{c}}$ but rather that such would be the case for a thermodynamically reversible nucleation process.

In the regular solution model the interaction parameter $A_{\mathrm{w}}$ is defined for $T_{\mathrm{c}}$ at $\zeta=0.5$ (Sect. 2.3). Thus to find $T_{\mathrm{c}}$ we look for the temperature that would produce a minimum in $\Delta G_{\text {het }}$ at $\zeta=0.5$. Mathematically, this is the temperature that simultaneously satisfies the conditions described in Eqs. (25) and (41). Figure 2 shows that this occurs around $T \sim 211 \mathrm{~K}$ for $\zeta=0.5$. Since both $\Delta G_{\text {het }}$ and $a_{\mathrm{w}, \text { eff }}$ depend on $T_{\mathrm{c}}$, we can iteratively solve Eqs. (36) and (46) to find $T_{\mathrm{c}}=211.473 \mathrm{~K}$. Figure 2 also suggests that when $T$ remains constant there is a critical value of $\zeta$ that marks the transition between two thermodynamic regimes. This is analyzed in Sect. 3.1.

\subsection{Kinetics of immersion freezing}

Almost every theoretical approach to describe the effect of INPs on ice formation focuses on the thermodynamics of ice nucleation. However as discussed in Sect. 1.1, increased molecular ordering increases the viscosity of vicinal water, implying that the immersed particle modifies the flux of water molecules to the nascent ice germ, hence the kinetics of ice nucleation (Etzler, 1983; Feibelman, 2010). Since these structural changes are also related to modifications in the chemical potential of the vicinal water, it is likely that the same mechanism that decreases $\Delta G_{\text {het }}$ also controls the mobility of water molecules in the environment around the particle. Such a connection between the water thermodynamic properties and its molecular mobility is well established (Adam and Gibbs, 1965; Debenedetti and Stillinger, 2001; Scala et al., 2000), but it is generally neglected in nucleation theory (e.g., Pruppacher and Klett, 1997; Ickes et al., 2017). In this section a heuristic model is proposed to account for such effects.

Kinetic effects modify the value of the impingement factor, $f_{\mathrm{het}}^{*}$, which controls the flux of water molecules to the ice germ. In general the ice germ grows by diffusion and rearrangement of nearby water molecules across the iceliquid interface, characterized by the interfacial diffusion coefficient, $D$. Increased ordering is characterized by a higher IL fraction, hence higher $\zeta$. Thus, in immersion freezing, $D$ must be a function of $\zeta$. Using Eq. (15) this can be expressed in the form

$f_{\text {het }}^{*}=\frac{\Omega}{v_{\mathrm{w}} d_{0}} D(\zeta)$.

Assuming that within the vicinal layer the ice germ grows following a similar mechanism as in the bulk of the liquid, then Eq. (16) can be applied to the heterogeneous process in the form

$D(\zeta)=D_{\infty}(\zeta)\left\{1+\exp \left[\frac{W_{\mathrm{d}}(\zeta)}{k_{B} T}\right]\right\}^{-1}$.

The last expression indicates that ice-liquid interfacial transfer requires a diffusional and a rearrangement component. $D_{\infty}(\zeta)$ characterizes purely diffusional processes occurring within the particle-liquid interface. Molecular rearrangement during ice germ growth within the vicinal layer is determined by $W_{\mathrm{d}}(\zeta)$. Since only molecules in the LL fraction of the vicinal water would rearrange to join the ice lattice then the latter is given by

$W_{\mathrm{d}}(\zeta)=W_{\mathrm{d}}(1-\zeta)=-n_{\mathrm{t}} \Delta \mu_{\mathrm{s}}(1-\zeta)$.

Introducing the last expression in Eq. (49) we obtain

$D(\zeta)=D_{\infty}(\zeta)\left[1+\exp \left(\frac{-n_{\mathrm{t}} \Delta \mu_{\mathrm{s}}(1-\zeta)}{k_{B} T}\right)\right]^{-1}$.

This expression is consistent with the thermodynamic model presented in Sect. 2.3, since as $\zeta$ increases, the vicinal water has a larger "ice" character, and fewer molecules need to rearrange to be incorporated into the growing ice germ.

\subsubsection{Diffusion within the particle-liquid interface}

The diffusional component of $D$ corresponds to the random jump of water molecules across the ice-liquid interface. For $\zeta \rightarrow 0$ there is no interaction between the particle and 
the adjacent water, hence diffusion must proceed as in the bulk of the supercooled water. At the opposite limit, $\zeta \rightarrow 1$ and $D_{\infty}(\zeta) \rightarrow 0$, which simply states that interfacial transfer vanishes when no net driving force exists across the iceliquid interface, i.e., the system is in equilibrium. To model this behavior the well-known relaxation theory proposed by Adam and Gibbs (1965) is employed (hereinafter, AG65). According to AG65, relaxation and diffusion in supercooled liquids require the formation of cooperative regions (CRs). The average transition probability and the timescale of diffusion are determined by the size of the smallest CR. Following a statistical mechanics treatment and assuming that each CR interacts weakly with the rest of the system, the authors derived the following expression for the average transition probability:

$\bar{W} \propto \exp \left(-\frac{A}{T S_{\mathrm{c}}}\right)$,

where $A$ represents the product of the minimum size of a $\mathrm{CR}$ in the liquid and the energy required to displace water molecules from their equilibrium position in the bulk, and $S_{\mathrm{c}}$ is the configurational entropy. Since $A$ is approximately constant, the mobility of water molecules is controlled by $S_{\mathrm{c}}$, which has been confirmed in molecular dynamics simulations and experimental studies (e.g., Scala et al., 2000; Debenedetti and Stillinger, 2001). The self-diffusivity of water is proportional to the transition probability and can be expressed in the form $D_{\infty} \sim D_{0} \bar{W}$, where $D_{0}$ is a constant. Using Eq. (52) this suggests the relationship

$$
\frac{D_{\infty}(\zeta)}{D_{\infty}}=\frac{\bar{W}(\zeta)}{\bar{W}(\zeta=0)}=\exp \left[-\frac{A}{T S_{\mathrm{c}, 0}}\left(\frac{S_{\mathrm{c}, 0}}{S_{\mathrm{c}}}-1\right)\right]
$$

where $D_{\infty}=D_{\infty}(\zeta=0)$ and $S_{\mathrm{c}, 0}=S_{\mathrm{c}}(\zeta=0)$ represent values in the bulk of the liquid. Equation (53) implies that the flux of molecules to the ice germ during immersion freezing is controlled by the configurational entropy of vicinal water. The usage of Eq. (53) thus requires developing an expression for $S_{\mathrm{c}}$, which is approximated in the form

$S_{\mathrm{c}}=(1-\zeta) S_{\mathrm{c}, \mathrm{LL}}+\zeta S_{\mathrm{c}, \mathrm{IL}}$

where $S_{\mathrm{c}, \mathrm{LL}}$ and $S_{\mathrm{c}, \text { IL }}$ are the configurational entropies of the LL and IL fractions, respectively. The term $S_{\mathrm{c}, \mathrm{LL}}$ in Eq. (54) dominates $S_{\mathrm{c}}$, since diffusion is controlled by molecules mobile enough to be incorporated in CRs (Stanley and Teixeira, 1980), although $S_{\mathrm{c}, \text { IL }}$ determines $S_{\mathrm{c}}$ when $\zeta \rightarrow 1$.

The regular model proposed in Sect. 2.3 suggests a weak interaction between IL and LL regions, since $\Delta G_{\text {mix }}$ is small compared to $\mu_{\mathrm{vc}}$. Thus we can approximate that $S_{\mathrm{c}, \mathrm{LL}} \approx S_{\mathrm{c}, 0}$. Unfortunately equating $S_{\mathrm{c} \text {,IL }}$ to the configurational entropy of bulk ice (which can be deduced from geometrical arguments; Pauling, 1935) would violate the requirement that $D \rightarrow 0$ at thermodynamic equilibrium. To estimate $S_{\mathrm{c}, \mathrm{IL}}$ we assume instead that water molecules in the IL regions should be displaced from their equilibrium position (essentially "diffusing" into the LL regions) to be incorporated into the ice lattice. During this process they gain an amount of energy equal to $-\Delta \mu_{\mathrm{s}}$ which is returned to the system upon entering the ice-liquid interface. Since this energy exchange results mostly from configurational rearrangement we can approximate that $S_{\mathrm{c}, \mathrm{IL}} \approx-\Delta \mu_{\mathrm{s}} / T$ (Barahona, 2014; Spaepen, 1975). With this, and using $\Delta \mu_{\mathrm{s}}=-k_{B} T \ln \left(\frac{a_{\mathrm{w}}}{a_{\mathrm{w}, \mathrm{eq}}}\right)$, Eq. (54) can be rewritten in the form

$S_{\mathrm{c}}=S_{\mathrm{c}, 0}(1-\zeta)+\zeta k_{B} \ln \left(\frac{a_{\mathrm{w}}}{a_{\mathrm{w}, \mathrm{eq}}}\right)$.

By introducing this expression into Eq. (53) and rearranging, we obtain

$\frac{D_{\infty}(\zeta)}{D_{\infty}}=\exp \left[-\frac{A}{T S_{\mathrm{c}, 0}} \frac{\zeta \sigma_{\mathrm{E}}}{\left(1-\zeta \sigma_{\mathrm{E}}\right)}\right]$,

where $\sigma_{\mathrm{E}}=1-S_{\mathrm{c}, 0}^{-1} k_{B} \ln \left(\frac{a_{\mathrm{w}}}{a_{\mathrm{w}, \mathrm{eq}}}\right)$. Using $D_{\infty}=D_{0} \bar{W}$, an equivalent expression to Eq. (56) can be written in the form

$D_{\infty}(\zeta)=D_{\infty}\left(\frac{D_{\infty}}{D_{0}}\right)^{\frac{\zeta \sigma_{\mathrm{E}}}{1-\zeta \sigma_{\mathrm{E}}}}$.

Equation (57) represents the effect of the immersed particle on the rate of growth of the ice germ. For $\zeta=0$, the particle does not affect the flux of water molecules to the nascent ice germ and $D_{\infty}(\zeta)=D_{\infty}$. However as $\zeta \rightarrow 1, D_{\infty}(\zeta) \propto$ $\exp \left(-\frac{1}{1-\zeta \sigma_{\mathrm{E}}}\right)$, and interface transfer becomes severely limited, particularly near equilibrium, since $\sigma_{\mathrm{E}} \rightarrow 1$. This effect is much stronger than the reduction in the dissipated work from an increased $\zeta$ (Sect. 2.5) and dominates $D$.

Introducing Eqs. (51) and (57) into Eq. (48) and rearranging, we obtain

$f_{\text {het }}^{*}=\frac{D_{\infty} \Omega}{v_{\mathrm{w}} d_{0}}\left(\frac{D_{\infty}}{D_{0}}\right)^{\frac{\zeta \sigma_{\mathrm{E}}}{1-\zeta \sigma_{\mathrm{E}}}}\left[1+\left(\frac{a_{\mathrm{w}}}{a_{\mathrm{w}, \mathrm{eq}}}\right)^{n_{\mathrm{t}}(1-\zeta)}\right]^{-1}$,

where $\Delta \mu_{\mathrm{s}}=-k_{B} T \ln \left(\frac{a_{\mathrm{w}}}{a_{\mathrm{w}, \mathrm{eq}}}\right)$ was used.

\subsection{Nucleation rate}

The results of Sects. 2.3 to 2.5 provide the basis for writing an expression for the ice nucleation rate of droplets by immersion freezing. Before completing such a description we need to provide an expression for $Z$. The application of Eq. (2) typically leads to the known expression (Pruppacher and Klett, 1997)

$Z=\left(\frac{\Delta G_{\text {het }}}{3 \pi k_{B} T n_{\text {het }}^{2}}\right)^{1 / 2}$.

On the other hand using Eq. (46) in Eq. (2), we obtain

$Z_{\mathrm{d}}=\left[\frac{\Delta G_{\mathrm{het}}\left(n_{\mathrm{het}}-2\right)^{1 / 3}}{3 \pi k_{B} T n_{\mathrm{het}}^{7 / 3}}\right]^{1 / 2}$, 
where the subscript "d" indicates that energy dissipation is taken into account. For $n_{\text {het }}>3$ it is easily verifiable that $Z_{\mathrm{d}} \approx Z$. Indeed the discrepancy between $Z_{\mathrm{d}}$ and $Z$ is only $30 \%$ for $n_{\text {het }}=3$, and it is much smaller for larger ice germs. However for $n_{\text {het }}=2, Z_{\mathrm{d}}=0$. This issue is rather fundamental and may represent the breaking of the assumption that each germ grows by the addition of a single molecule at a time. Hence Eq. (59) will be used keeping in mind that for very small ice germs, it represents only an approximation.

With the above considerations it is now possible to substitute Eqs. (46), (47), (58) and (59) into Eq. (5) to obtain the heterogeneous ice nucleation rate

$$
\begin{aligned}
J_{\text {het }} & =\frac{2 Z D_{\infty} \Omega}{3 v_{\mathrm{w}}^{2}}\left(\frac{D_{\infty}}{D_{0}}\right)^{\frac{\zeta \sigma_{\mathrm{E}}}{1-\zeta \sigma_{\mathrm{E}}}}\left[1+\left(\frac{a_{\mathrm{w}}}{a_{\mathrm{w}, \mathrm{eq}}}\right)^{n_{\mathrm{t}}(1-\zeta)}\right]^{-1} \\
& \exp \left(-\frac{\left.n_{\mathrm{het}} \Delta \mu_{\mathrm{i}}\right|_{a_{\mathrm{w}, \text { eff }}}}{2 k_{B} T}\right)
\end{aligned}
$$

where $d_{0}=\left(6 v_{\mathrm{w}} / \pi\right)^{1 / 3}$ and $a_{0}=\pi d_{0}^{2} / 4$ were used, and $\Omega=$ $\Gamma_{\mathrm{w}} s n_{\text {het }}^{2 / 3} a_{0}$ is the surface area of the ice germ. Other symbols and values used are listed in Appendix A.

\subsection{The role of active sites}

There is evidence that in dust and other INPs, ice is formed preferentially in the vicinity of surface patches, commonly referred as active sites. The existence of active sites has been established experimentally for deposition ice nucleation (i.e., ice nucleation directly from the vapor phase; Kiselev et al., 2017), and they may be also important for immersion freezing (e.g., Murray et al., 2012). In the classical view active sites have the property of locally reducing $\Delta G_{\text {het }}$, increasing $J_{\text {het }}$. In the so-called singular hypothesis each active site has an associated characteristic temperature at which it nucleates ice. Current interpretation assigns $J_{\text {het }} \rightarrow \infty$ at each active site at its characteristic temperature, with some variability due to "statistical fluctuations" in the germ size (Vali, 2014). Some CNT-based approaches to describe immersion freezing account for the existence of active sites by assuming a distribution of contact angles for each particle. Hence each active site is assigned a characteristic contact angle instead of a characteristic temperature (e.g., Zobrist et al., 2007; Ickes et al., 2017).

The view of the role of active sites as capable of locally decreasing $\Delta G_{\text {het }}$ relies heavily on an interpretation of immersion freezing that mimics ice nucleation from the vapor phase (Fig. 3a). Such a description is, however, too limited for ice formation within the liquid phase. For example, it is implicitly assumed that the active site brings molecules together, similar to an adsorption site. However a particle immersed within a liquid is already surrounded by water molecules (Fig. 3b). In fact, nascent ice structures are associated with low-density regions within the liquid (Bullock and Molinero, 2013). Thus in the classical view the active site should be
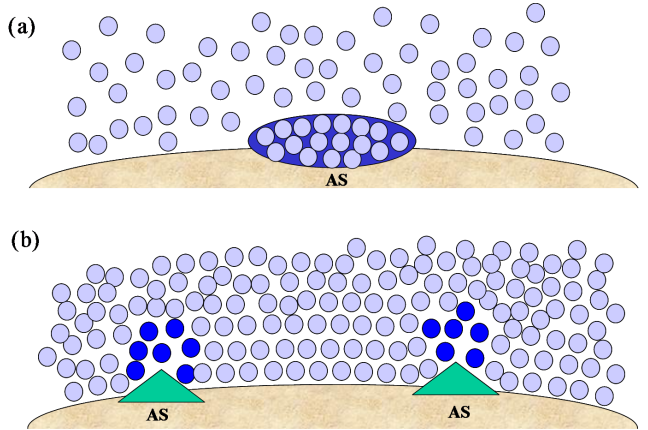

Figure 3. Different representations of immersion freezing. (a) an ice germ (dark blue) forming on an active site (AS) by random collision of water molecules (light blue). (b) low-density regions (dark blue) forming in the vicinity of active sites within a dense liquid phase (light blue).

able to "pull molecules apart" instead of bringing them together. This creates a conceptual problem. To locally reduce $\Delta G_{\text {het }}$ active sites should be able to permanently create lowdensity regions within the liquid, which would require a large amount of energy. In other words, active sites would have the unusual property of creating a thermodynamic barrier maintaining their surrounding water in a non-equilibrium state. Such situation is unlikely in immersion freezing.

The concept of a local nucleation rate also presents some difficulties. In the strict sense $J_{\text {het }}$ is the velocity with which the size distribution of molecular clusters in an equilibrium population crosses the critical size (Kashchiev, 2000; Seinfeld and Pandis, 1998). In immersion freezing the domain of such a distribution is the whole volume of the droplet. Thus only a single value of $J_{\text {het }}$ can be defined for a continuous liquid phase, independently of where the actual nucleation process is occurring, since no permanent spatial gradients of $T$ or concentration exist within equilibrium systems. Having otherwise implies that parts of the system would need to be maintained in a non-equilibrium state, having their own cluster size distribution. This requires the presence of non-permeable barriers within the liquid, a condition not encountered in immersion freezing. Similarly, the characteristic temperature of an active site is an unmeasurable quantity, since a system in equilibrium has the same temperature everywhere. Hence it would be impossible to distinguish whether the particle as a whole or only the active site must reach a certain temperature before nucleation takes place.

These difficulties can be reconciled if, instead of promoting nucleation through a thermodynamic mechanism, active sites provide a kinetic advantage to ice nucleation. A way in which this can be visualized is shown in Fig. 3b. The vicinal water is in equilibrium with the particle and exhibits a larger degree of ordering near the interface. Since in immersion freezing the formation of ice in the liquid depends on molecular rearrangement, the active site should produce a transient structural transformation that allows the propa- 
gation of ice. These sites would be characterized by defects where templating is not efficient, allowing greater molecular movement, hence facilitating restructuring. Their presence is guaranteed, since particles are never uniform at the molecular scale. In this view active sites create ice by promoting fluctuation instead of by locking water molecules in strict configurations. It implies that for uniform systems (e.g., a single droplet with a single particle) $\Delta G_{\text {het }}$ depends on the equilibrium between the particle and the vicinal water, and active sites enhance fluctuation around specific locations. This obviates the need for the hypothesis of a well-defined characteristic temperature for each active site. It, however, does not mean that active sites are transient. They are permanent features of the particle and should have a reproducible behavior, inducing ice nucleation around the same place in repeated experiments (e.g., Kiselev et al., 2017).

Within the framework presented above, there can only be one $J_{\text {het }}$ defined in the droplet volume. The presence of active sites introduces variability in $J_{0}$ instead of $\Delta G_{\text {het }}$. The latter is determined by the thermodynamic equilibrium between the particle and its vicinal water. Although the theory presented here does not account for internal gradients in the droplet-particle system, in practice it is likely that the observed $J_{\text {het }}$ corresponds to the site promoting the largest density fluctuations. Variability in $J_{\text {het }}$ would be introduced by fluctuation in the cluster size distribution in the liquid and from the multiplicity of active sites in the particle population. In this sense the proposed view is purely stochastic.

\section{Discussion}

\subsection{Ice nucleation regimes}

A consequence of the linkage between the properties of vicinal water and $\Delta G_{\text {het }}$ is the existence of distinct nucleation regimes. This was mentioned in Sect. 2.4.1, and here it is explored in detail. Recall from Fig. 2 that for a given temperature, $\Delta G_{\text {het }}$ passes by a minimum defined by the condition $\frac{\partial^{2} \Delta G_{\text {het }}}{\partial n_{\text {het }}^{2}}=0$. Figure $4 \mathrm{~b}$ depicts a similar behavior when varying $T$. It shows that for a given $\zeta$ there is a temperature $T_{\mathrm{S}}$ at which $\Delta G_{\text {het }}$ is minimum. For $T>T_{\mathrm{s}}, \Delta G_{\text {het }}$ increases with increasing $T$ because $n_{\text {het }}$ increases (Fig. $4 \mathrm{a}$ ). This is the typical behavior predicted by the classical model (e.g., Khvorostyanov and Curry, 2005), hence such regime will be termed "germ-forming", since $\Delta G_{\text {het }}$ is determined by the formation of the ice-liquid interface.

A different behavior is found for $T<T_{\mathrm{s}}$, where $\Delta G_{\text {het }}$ decreases with increasing $T$. In this regime $n_{\text {het }}$ remains almost constant at very low values, $\Delta G_{\text {het }}$ is small and results mostly from the dissipation of work. Ice nucleation is not limited by the formation of the ice-liquid interface but rather by the propagation of small fluctuations in the vicinity of preformed ice-like regions. Therefore it is controlled by the diffusion of water molecules to such regions rather than by $\Delta G_{\text {het }}$. This is
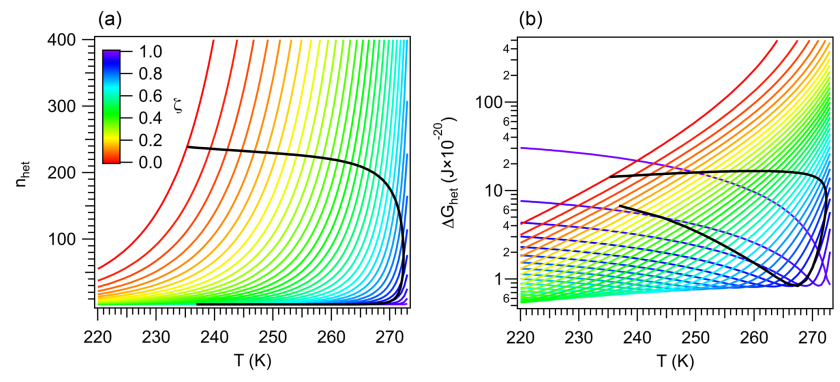

Figure 4. Critical germ size (a) and work of heterogeneous ice nucleation (b) for different values of $\zeta$ (color). Black lines correspond to constant $J_{\text {het }}=10^{6} \mathrm{~m}^{-2} \mathrm{~s}^{-1}$.

akin to a spinodal decomposition process (Cahn and Hilliard, 1958) and will be termed "spinodal ice nucleation". It is, however, not truly spinodal decomposition, since it requires a finite, albeit small, amount of work to occur.

Since for each value of $\zeta$ there is a minimum in $\Delta G_{\text {het }}$ (Fig. 4), theoretically all INPs are capable of nucleating ice in both regimes. In practice spinodal ice nucleation would only occur if $T_{\mathrm{S}}$ lies within the $233 \mathrm{~K}<T<273 \mathrm{~K}$ range, where immersion freezing occurs. For example, for $\zeta=0.1$, Fig. 4b shows that the minimum in $\Delta G_{\text {het }}$ occurs at $T<220 \mathrm{~K}$. Since homogeneous ice nucleation should occur above this temperature, INPs characterized by $\zeta=0.1$ will not exhibit spinodal ice nucleation. These particles always nucleate ice in the classical germ-forming regime $\left(T>T_{\mathrm{S}}\right)$. The situation is, however, different for $\zeta=0.9$, since $T_{\mathrm{s}} \approx 270 \mathrm{~K}$. These INPs are capable of nucleating ice in both the spinodal $\left(T<T_{\mathrm{S}}\right)$ and the germ-forming $\left(T>T_{\mathrm{S}}\right)$ regimes. For the spinodal regime, $\Delta G_{\text {het }}$ is low and decreases slightly with increasing $T$, indicating that the thermodynamic barrier to nucleation is virtually removed. Ice formation is therefore almost entirely controlled by kinetics.

The existence of the spinodal nucleation regime signals the possibility of an interesting behavior in freezing experiments, where the same $\Delta G_{\text {het }}$ may correspond to two very different INPs. To show this the values of $\Delta G_{\text {het }}$ and $n_{\text {het }}$ corresponding to $J_{\text {het }}=10^{6} \mathrm{~m}^{-2} \mathrm{~s}^{-1}$ are depicted in Fig. 4 with black lines. These lines form semi-closed curves when plotted against temperature indicating that the same $\Delta G_{\text {het }}$ may correspond to two different values of $\zeta$. The upper branch (with high $\Delta G_{\text {het }}$ ) corresponds to the germ-forming regime and the lower branch to the spinodal regime. This picture may be convoluted by the fact that high $\zeta$ also implies strong kinetic limitations during ice nucleation and is further discussed in Sect. 3.3.

\subsection{Pre-exponential factor}

Kinetic effects on ice nucleation are typically analyzed in terms of the pre-exponential factor, which is proportional to 


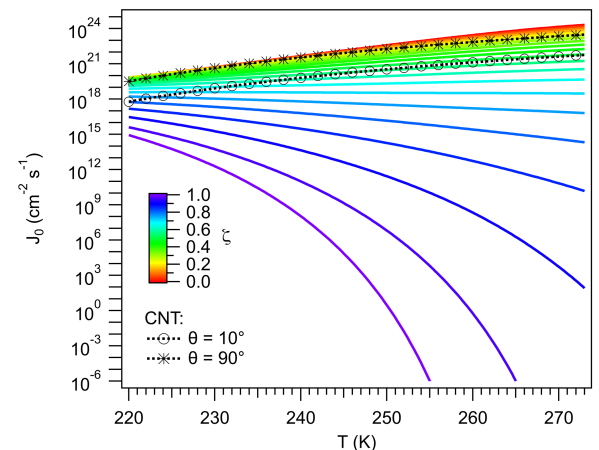

Figure 5. Pre-exponential factor. Colored lines indicate different values of $\zeta$. Black lines correspond to results calculated using CNT for different values of the contact angle, $\theta$.

$f_{\text {het }}^{*}$ in the form

$J_{0}=\frac{Z f_{\text {het }}^{*}}{a_{0}}$.

$J_{0}$ expresses the normalized flux of water molecules to the ice germ, corrected by $Z$. Figure 5 shows $J_{0}$ calculated using Eqs. (58) and (59). Results from CNT (Eq. 6) are also shown. In general $J_{0}$ varies with $T$ and $\zeta$. The sensitivity of $J_{0}$ to $T$ is determined by $D_{\infty}$ (Barahona, 2015), with $J_{0}$ increasing with $T$, since water molecules increase their mobility. Also, at higher $T$, less work is dissipated during interface transfer. These effects dominate the variation in $J_{0}$ for $\zeta<0.5$, suggesting that the particle has a limited effect on the mobility of vicinal water. Ice nucleation around these particles would be reasonably well described by assuming a negligible effect of the particle on $J_{0}$, as done in CNT. This is evidenced by the CNT-derived values for $\theta=10^{\circ}$ and $\theta=90^{\circ}$, which represent particles with high and low particle-ice affinity, respectively, and correspond to the range of expected variability in CNT. The $\theta=90^{\circ}$ and $\zeta \sim 0$ lines in Fig. 5 are within 1 order of magnitude of each other and are in agreement with homogeneous nucleation results (Barahona, 2015). The $\theta=10^{\circ}$ line is also close to the $\zeta \sim 0.5$ curve. In both cases $J_{0}$ increases by about 2 orders of magnitude between $220 \mathrm{~K}$ and $273 \mathrm{~K}$ and decreases by about 2 orders of magnitude from $\zeta=0.0$ to $\zeta=0.5$, or from $\theta=90^{\circ}$ to $\theta=10^{\circ}$ in CNT. This reflects the effect of variation in $Z$ on $J_{0}$.

The behavior of $J_{0}$ for $\zeta>0.5$ dramatically differs from CNT. For $\zeta>0.5$, and particularly for $\zeta>0.8, J_{0}$ decreases strongly with increasing $T$. This is because as $\zeta \rightarrow 1$ and $T \rightarrow 273 \mathrm{~K}$, the driving force for interfacial transfer, i.e., the separation of $\mu_{\mathrm{vc}}$ from thermodynamic equilibrium, vanishes. As the system moves near these conditions $D$ becomes very small. This is the result of the high IL fraction of the vicinal water limiting the number of configurations available to form cooperative regions, required to induce water mobility (Sect. 2.5.1). Such behavior cannot be reproduced by $\mathrm{CNT}$, since no explicit dependency of $D$ on the properties of the vicinal layer is accounted for. For $\zeta>0.99 J_{0}$ decreases by more than 30 orders of magnitude from $220 \mathrm{~K}$ to $273 \mathrm{~K}$; molecular transport nearly stops. Ice nucleation may not be possible at such an extreme, despite the fact that these particles very efficiently reduce $\Delta G_{\text {het }}$ (Fig. 4); water may remain in the liquid state at very low temperatures. Such an effect has been experimentally observed in some biological systems (Wolfe et al., 2002).

\subsection{Nucleation rate}

The interplay between kinetics and thermodynamics determines the complex behavior of $J_{\text {het }}$ in immersion ice nucleation. Particles highly efficient at decreasing $\Delta G_{\text {het }}$ also decrease the rate of interfacial diffusion to the point where they may effectively prevent ice nucleation. On the other hand, INPs with low $\zeta$ do not significantly affect $J_{0}$ but have a limited effect on $\Delta G_{\text {het }}$. This is confounded with the presence of two thermodynamic nucleation regimes, one in which $\Delta G_{\text {het }}$ may be large and increases with $T$ ("germ-forming"), and another in which $\Delta G_{\text {het }}$ is very small and decreases as $T$ increases ("spinodal nucleation"). This picture can be simplified, since within the range $233 \mathrm{~K}<T<273 \mathrm{~K}$, where immersion freezing is relevant for atmospheric conditions, INPs with $\zeta>0.7$ are, at the same time, more likely to nucleate ice in the spinodal regime and to exhibit strong kinetic limitations. Similarly for $\zeta<0.6$ the transition to spinodal nucleation occurs below $233 \mathrm{~K}$ (Fig. 2). These INPs tend to nucleate ice in the germ-forming regime without significantly affecting $J_{0}$. Thus the thermodynamic regimes introduced in Sect. 3.1 loosely correspond to kinetic regimes. Roughly, ice nucleation in the spinodal regime is controlled by kinetics, and in the germ-forming regime, it is controlled by thermodynamics. This is a useful approximation, but it should be used with caution. Even in the germ-forming regime the particle affects the kinetics of ice-liquid interfacial transfer to some extent. Similarly, in the spinodal regime $\Delta G_{\text {het }}$ is small, but finite.

Figure 6 shows the behavior of $J_{\text {het }}$ as $T$ increases for different values of $\zeta$. $J_{\text {het }}$ in the germ-forming regime resembles the behavior predicted by CNT. $J_{\text {het }}$ increases steeply with decreasing $T$ and increasing $\zeta$. Similarly for CNT, $J_{\text {het }}$ increases for decreasing $T$ and $\theta$. This is characteristic of the thermodynamic control on $J_{\text {het }}$, where $\Delta G_{\text {het }}$ and $\frac{\mathrm{d} \Delta G_{\text {het }}}{\mathrm{d} T}$ are large (Fig. 4), and $J_{0}$ is relatively unaffected by the particle. In this regime it is always possible to find a contact angle (typically between 10 and $100^{\circ}$ ) that results in close agreement of $J_{\text {het }}$ between CNT and NNF predictions (Fig. 6), particularly for $J_{\text {het }}<10^{12} \mathrm{~cm}^{-2} \mathrm{~s}^{-1}$, which covers most values of atmospheric interest. This is also true for $a_{\mathrm{w}}=0.9$ (Fig. 6), although the approximation to the equilibrium temperature signals a steeper behavior in CNT peaking at higher values than NNF. Since $\frac{\mathrm{d} J_{\text {het }}}{\mathrm{d} T}$ is large, $J_{\text {het }}$ may show threshold behavior, characteristic of ice nucleation mediated by some dust species like Chlorite and Montmorillonite (Atkin- 

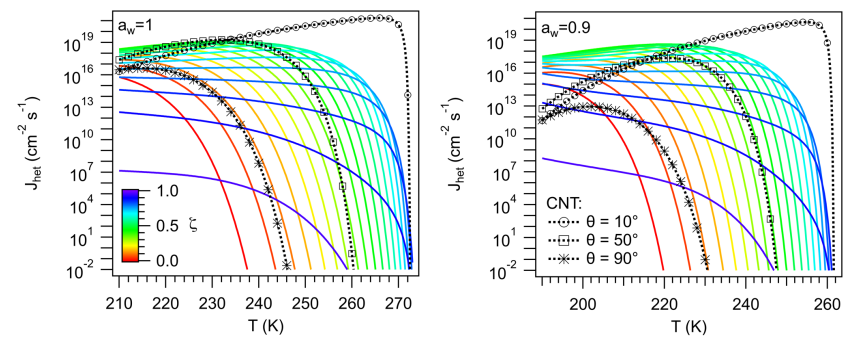

Figure 6. Ice nucleation rate calculated using Eq. (61) for different values of $\zeta$ (color). Black lines were calculated using CNT for different values of the contact angle, $\theta$.

son et al., 2013; Murray et al., 2012; Hoose and Möhler, 2012).

There is, however, no value of $\theta$ that would lead to overlap between CNT and NNF for $\zeta>0.7$. These conditions largely correspond to spinodal ice nucleation. $J_{\text {het }}$ is kinetically controlled, since $\Delta G_{\text {het }}$ is small, and $J_{0}$ varies widely with $T$ (Fig. 5). As in the germ-forming regime $J_{\text {het }}$ also reaches significant values but increases more slowly with decreasing $T$ (Fig. 6). Higher $\zeta$ leads to $J_{\text {het }}$ becoming significant at higher $T$. But unlike in the germ-forming case, curves with higher $\zeta$ tend to plateau at progressively lower values of $J_{\text {het }}$, since they become kinetically limited by their approximation to the thermodynamic equilibrium. For $\zeta \sim 0.7$ some of the curves of Fig. 6 also display germ-forming behavior at high $T$ and are characterized by a sudden decrease in $-\frac{\mathrm{d} J_{\text {het }}}{\mathrm{d} T}$ as $T$ decreases. The sudden change of slope corresponds to the region around the minimum $\Delta G_{\text {het }}$ (Fig. 4) and signals the transition from germ-forming to spinodal ice nucleation. Such behavior has been observed in some INPs of bacterial origin (Murray et al., 2012).

Figure 6 also indicates that nucleation regimes cannot be assigned based on the values of $J_{\text {het }}$ or on the observed freezing temperature, $T_{\mathrm{f}}$. In both regimes, $J_{\text {het }}$ may reach substantial values, hence $T_{\mathrm{f}}$ may cover the entire range $233 \mathrm{~K}<T<$ $273 \mathrm{~K}$. What is striking is that $J_{\text {het }}$ curves with $\zeta>0.7$ tend to cross those with $\zeta<0.7$. This means that two INPs characterized by very different $\zeta$ can have the same freezing temperature. This result thus challenges the common notion that INPs with higher freezing temperatures are intrinsically more active at nucleating ice, or in other words, that by measuring $T_{\mathrm{f}}$ alone, it is possible to characterize the freezing properties of a given material. In reality, to discern whether the observed $T_{\mathrm{f}}$ corresponds to a good (in the thermodynamic sense) INP acting in the spinodal regime or a less active INP acting in the germ-forming regime, it is necessary to measure $\frac{\mathrm{d} J_{\text {het }}}{\mathrm{d} T}$ along with $T_{\mathrm{f}}$.

\subsection{Application to the water activity-based nucleation rate}

If a droplet is in equilibrium with its environment then $a_{\mathrm{w}}$ is a function of the relative humidity. Thus the relationship between $a_{\mathrm{w}}$ and the freezing temperature, $T_{\mathrm{f}}$, conveys important information about the potential of a particle to catalyze the formation of ice and can be used to generate parameterizations of immersion ice nucleation for cloud models (Kärcher and Lohmann, 2003; Koop and Zobrist, 2009; Barahona and Nenes, 2009). A widely used class of parameterizations is based on the so-called water activity criterion (Koop et al., 2000; Koop and Zobrist, 2009), the condition that for a given material the water activity at which heterogeneous ice nucleation is observed, $a_{\mathrm{w}, \text { het }}$, is related by a constant to $a_{\mathrm{w}, \text { eq }}$ (Koop et al., 2000; Koop and Zobrist, 2009). Here it is shown that the two-state thermodynamic model proposed in Sect. 2.3 implies the water activity criterion as a purely thermodynamic constraint to freezing.

\subsubsection{Water activity shift}

By definition the thermodynamic path shown in Fig. 1 operates between two equilibrium states. The relation between $\Delta G_{\text {het }}$ and $\Delta G_{\text {hom }}$ is therefore independent of the way the system reaches $a_{\mathrm{w}, \text { eff. In the absence of any kinetic limita- }}$ tions to the germ growth, Eq. (35) also represents a direct relationship between $J_{\text {hom }}$ and $J_{\text {het }}$. (Kärcher and Lohmann, 2003; Marcolli et al., 2007; Koop and Zobrist, 2009; Knopf and Alpert, 2013). Thus one can imagine two separate experiments in which the environmental conditions are set to either $a_{\mathrm{w}}$ or $a_{\mathrm{w}, \text { eff }}$, the former resulting in heterogeneous freezing and the latter in homogeneous ice nucleation. Under these conditions Eq. (34) implies that when heterogeneous ice nucleation is observed at $a_{\mathrm{w}, \text { het }}=a_{\mathrm{w}}$ there is a corresponding homogeneous process that would occur at $a_{\mathrm{w}, \mathrm{hom}}=a_{\mathrm{w} \text {,eff. }}$. Thus we can write an equivalent expression to Eq. (34), but in terms of $a_{\mathrm{w}, \text { het }}$ and $a_{\mathrm{w}, \text { hom }}$, in the form

$a_{\mathrm{w}, \text { het }}=a_{\mathrm{w}, \mathrm{hom}}\left(\frac{a_{\mathrm{w}, \mathrm{eq}}}{a_{\mathrm{w}, \mathrm{hom}}}\right)^{\zeta} \exp \left(\Lambda_{\mathrm{mix}}\right)$.

Eq. (63) can be rewritten as

$\ln \left(a_{\mathrm{w}, \mathrm{het}}\right)=(1-\zeta) \ln \left(a_{\mathrm{w}, \mathrm{hom}}\right)+\zeta \ln \left(a_{\mathrm{w}, \mathrm{eq}}\right)+\Lambda_{\text {mix }}$.

Subtracting $\ln \left(a_{\mathrm{w}, \text { eq }}\right)$ from each side of Eq. (64) gives

$$
\begin{aligned}
& \ln \left(a_{\mathrm{w}, \text { het }}\right)-\ln \left(a_{\mathrm{w}, \mathrm{eq}}\right)= \\
& \quad(1-\zeta)\left[\ln \left(a_{\mathrm{w}, \text { hom }}\right)-\ln \left(a_{\mathrm{w}, \text { eq }}\right)\right]+\Lambda_{\text {mix }} .
\end{aligned}
$$

Using the approximation $\ln (x) \approx x-1$ for $x \sim 1$, Eq. (65) can be linearized in the form

$\Delta a_{\mathrm{w}, \text { het }}=\Delta a_{\mathrm{w}, \text { hom }}(1-\zeta)+\Lambda_{\text {mix }}$,

where $\Delta a_{\mathrm{w}, \text { hom }}=a_{\mathrm{w}, \mathrm{hom}}-a_{\mathrm{w}, \mathrm{eq}}$ and $\Delta a_{\mathrm{w}, \text { het }}=a_{\mathrm{w}, \text { het }}-a_{\mathrm{w}, \mathrm{eq}}$ are the homogeneous and heterogeneous water activity shifts, 
respectively. $\Delta a_{\mathrm{w}, \text { hom }}$ has been found to be approximately constant for a wide range of solutes (Koop et al., 2000); therefore Eq. (66) suggests that $\Delta a_{\mathrm{w} \text {,het }}$ should be approximately constant, since $\Lambda_{\text {mix }} \sim 0.02$ and only depends on $T$. Thus, the two-state model presented in Sect. 2.3 implies the so-called water activity criterion (Koop et al., 2000) for heterogeneous ice nucleation, giving support to the hypothesis that increasing order near the particle surface drives ice nucleation.

Equations (63) to (66) are fundamental thermodynamic relationships of the system and can be used to analyze the effect of the immersed particle on ice formation independently of kinetic effects. To do so $a_{\mathrm{w} \text {,hom }}$ must be determined entirely by thermodynamics. This is because if $a_{\mathrm{w}, \text { hom }}$ is defined at some $J_{\text {hom }}$ threshold then it (and by extension $a_{\mathrm{w}, \text { het }}$ ) would also depend on the freezing kinetics. Fortunately, a thermodynamic definition of $a_{\mathrm{w}, \text { hom }}$ has been achieved by Baker and Baker (2004). The authors showed that, on average, freezing occurs below the temperature at which the compressibility of water reaches a maximum. At this point density fluctuations are wide enough to allow for structural transformations that facilitate the formation of ice-like regions within the droplet volume. Such a criterion does not depend on measured freezing rates and can be extended to the freezing of water solutions, coinciding with the results of Koop et al. (2000). Bullock and Molinero (2013) also derived a pure thermodynamic criterion for $a_{\mathrm{w}, \text { hom }}$ using the equilibrium between low-density regions and the bulk solution. Within these frameworks $a_{\mathrm{w} \text {,hom }}$ can be defined without reference to a $J_{\text {hom }}$ threshold. By extension, Eq. (64) guarantees that $a_{\mathrm{w}, \text { het }}$ can be determined entirely by the thermodynamic properties of the system.

Equation (64) also implies that for a given $a_{\mathrm{w}, \text { hom }}$ there is a temperature for which $a_{\mathrm{w}}=a_{\mathrm{w} \text {, het }}$, referred as the "thermodynamic freezing temperature", $T_{\mathrm{ft}}$. Formally, $T_{\mathrm{ft}}$ represents the solution of

$$
\begin{aligned}
\ln \left(a_{\mathrm{w}}\right) & -(1-\zeta) \ln \left[a_{\mathrm{w}, \mathrm{eq}}\left(T_{\mathrm{ft}}\right)+\Delta a_{\mathrm{w}, \mathrm{hom}}\right]-\zeta \ln \left[a_{\mathrm{w}, \mathrm{eq}}\left(T_{\mathrm{ft}}\right)\right] \\
& -\Lambda_{\text {mix }}\left(T_{\mathrm{ft}}\right)=0,
\end{aligned}
$$

or in the linearized form,

$a_{\mathrm{w}}-a_{\mathrm{w}, \mathrm{eq}}\left(T_{\mathrm{ft}}\right)-\Delta a_{\mathrm{w}, \mathrm{hom}}(1-\zeta)-\Lambda_{\text {mix }}\left(T_{\mathrm{ft}}\right)=0$.

Since $\Delta a_{\mathrm{w}, \text { hom }}$ is considered a thermodynamic property of the system (Baker and Baker, 2004), $T_{\mathrm{ft}}$ does not depend on the freezing kinetics. Thus $T_{\mathrm{ft}}$ can be interpreted as the highest temperature where it is likely to observe ice nucleation for a given thermodynamic state (determined by $a_{\mathrm{w}}, \zeta$ and the system pressure).

Figure 7 shows the $T_{\mathrm{ft}}-a_{\mathrm{w}}$ relationship defined by Eq. (67), calculated using $\Delta a_{\mathrm{w} \text {,hom }}=0.304$ (Koop et al., 2000; Barahona, 2014; Baker and Baker, 2004). As expected, the figure resembles experimental results found by several authors (e.g., Koop and Zobrist, 2009; Zuberi et al., 2002; Zobrist et al., 2008; Alpert et al., 2011; Knopf and Alpert, 2013),

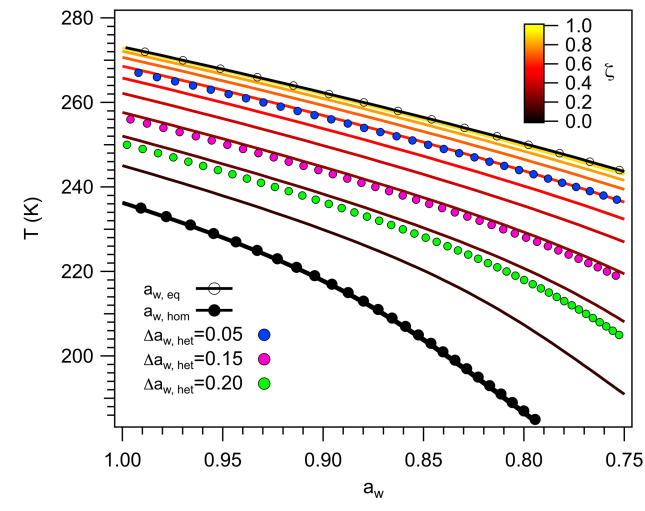

Figure 7. Thermodynamic freezing temperature as a function of water activity. Colored lines correspond to $T_{\mathrm{ft}}\left(a_{\mathrm{w}}=a_{\mathrm{w} \text {, het }}\right)$ for different values of $\zeta$. Also shown are the water activities at equilibrium and at the homogeneous freezing threshold, $a_{\mathrm{w}, \text { eq }}$ and $a_{\mathrm{w}, \text { hom }}$, respectively, and lines drawn applying constant water activity shifts, $\Delta a_{\mathrm{w}, \text { het }}$, of $0.05,0.15$ and 0.20 .

where curves for $\zeta>0$ align with constant water activity shifts to $a_{\mathrm{w}, \text { eq }}$. To make this evident, lines were drawn using constant values of $\Delta a_{\mathrm{w}, \text { het }}=0.05,0.15$ and 0.20 , which coincide with lines corresponding to $\zeta=0.2,0.3$ and 0.7 , respectively. This shows that Eq. (66) is a good approximation to Eq. (63) and constitutes a theoretical derivation of the water activity criterion. The fact that such behavior can be reproduced by Eq. (63) validates the regular solution approximation used in Sect. 2.3 and supports the idea that the effect of the immersed particle on ice nucleation can be explained as a relative increase in the ice-like character of the vicinal water.

It must be emphasized that $T_{\mathrm{ft}}$ only establishes the potential of an INP to induce freezing at $a_{\mathrm{w}}=a_{\mathrm{w} \text {,het, }}$ regardless of whether a measurable $J_{\text {het }}$ can be experimentally realized. Physically, it is plausible that as the particle increases the icelike character of the vicinal water, it also increases the probability of wide density fluctuations. As a result low-density regions, wide enough to accommodate the ice gem, exist at higher $T$ than in homogeneous ice nucleation. Following the argument of Baker and Baker (2004) this would also imply that the compressibility of water near the particle reaches a maximum at higher $T$ than in the bulk. More research however is needed to elucidate this point. The presence of a spinodal regime would also mean that the observed freezing temperature may differ from $T_{\mathrm{ft}}$, since at such a limit nucleation, it is no longer controlled by thermodynamics. This is illustrated in the next section.

\subsubsection{Freezing by humic-like INPs}

$\Delta a_{\mathrm{w}, \text { het }}$ has been determined in several studies and has been used to predict and parameterize $J_{\text {het }}$ in atmospheric models (e.g., Zobrist et al., 2008; Knopf and Alpert, 2013). Thus it 
is useful in analyzing the conditions under which $\zeta$ (hence

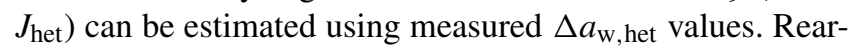
ranging Eq. (66) we obtain

$\Delta a_{\mathrm{w}, \text { het }}-\Delta a_{\mathrm{w}, \mathrm{hom}}(1-\zeta)-\Lambda_{\mathrm{mix}}=0$.

If $\Delta a_{\mathrm{w}, \text { hom }}$ and $\Delta a_{\mathrm{w} \text {, het }}$ are known, $\zeta$ can be estimated iteratively by solving Eq. (69). Note that $\Lambda_{\text {mix }}$ is temperature dependent (Eq. 34), implying a slight dependency of $\zeta$ on $T$ when $\Delta a_{\mathrm{w} \text {, het }}$ is constant. However since $\Lambda_{\text {mix }}$ is also typically small, $\zeta$ is almost equal to $1-\frac{\Delta a_{\mathrm{w}, \mathrm{het}}}{\Delta a_{\mathrm{w} \text { hom }}}$.

To test Eq. (69) the data for leonardite (LEO) and Pahokee peat (PP) particles (humic-like substances) obtained by Rigg et al. (2013) are used. The authors reported $\Delta a_{\mathrm{w}, \text { het }}=0.2703$ for LEO and $\Delta a_{\mathrm{w}, \text { het }}=0.2466$ for PP. These values are assumed to be independent of $a_{\mathrm{w}}$ and $T$, with an experimental error in $\Delta a_{\mathrm{w}, \text { het }}$ of 0.025 . The average $J_{\text {het }}$ obtained from different samples and from repeated freezing and melting experiments for both materials is depicted in Fig. 8. Applying Eq. (69) over the $T=210 \mathrm{~K}-250 \mathrm{~K}$ range and using $\Delta a_{\mathrm{w}, \text { hom }}=0.304$ results in $\zeta=0.049-0.058$ for LEO and $\zeta=0.096-0.121$ for PP. Within this temperature range these values correspond to the germ-forming regime, hence $J_{\text {het }}$ is thermodynamically controlled. A comparison against the experimentally determined $J_{\text {het }}$ for three different values of $a_{\mathrm{w}}$ is shown in Fig. 8. Within the margin of error there is a reasonable agreement between the modeled and the experimental $J_{\text {het. }}$.

The top panels of Figure 8, however, reveal that even if $J_{\text {het }}$ becomes significant around the values predicted by Eq. (69), $-\frac{\mathrm{d} \ln J_{\text {het }}}{\mathrm{d} T}$ is overestimated, particularly for PP. This may indicate that these INPs nucleate ice in the spinodal regime. To test this hypothesis $J_{\text {het }}$ was fitted to the reported measurements by varying $\zeta$ within the range where spinodal nucleation would be dominant. To avoid agreement by design a single $\zeta$ was used for all experiments for each species resulting in $\zeta=0.949$ for PP and $\zeta=0.952$ for LEO (Fig. 8, bottom panels). For PP, $J_{\text {het }}$ and $-\frac{\mathrm{d} \ln J_{\text {het }}}{\mathrm{d} T}$ agree better with the experimental values, whereas for LEO the agreement improves at high $T$ but worsens at low $T$. In this regime $J_{\text {het }}$ seems to be slightly overestimated by the theory at the lowest $a_{\mathrm{w}}$ tested. This may be due to small uncertainties in $a_{\mathrm{w}}$ that play a large role in $J_{\text {het }}$ (for example, the assumption of a $T$-independent $a_{\mathrm{w}}$; Alpert et al., 2011). There is the possibility that the humic acid present in PP may slightly dissolve during the experiments (Daniel Knopf, personal communication, 2017), which would impact not only $a_{\mathrm{w}}$ but also may modify the composition of the particles, hence $\zeta$.

The exercise above suggests that ice nucleation in PP may follow a spinodal mechanism. Using a single value of $\Delta a_{\mathrm{w}, \text { het }}$ to predict $\zeta$, as expressed mathematically by Eq. (69), seems to work for LEO. Since Eq. (69) represents a thermodynamic relation between $\Delta a_{\mathrm{w}, \text { hom }}$ and $\Delta a_{\mathrm{w} \text {, het }}$, it is expected to work well when nucleation is thermodynamically controlled, i.e., the germ-forming regime. However it (a)
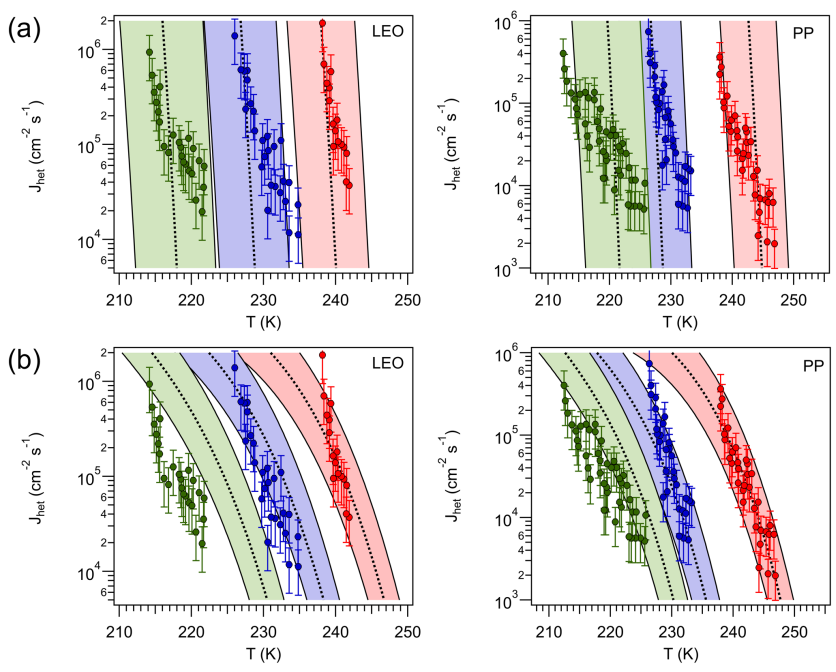

Figure 8. (a) Heterogeneous ice nucleation rate calculated using a constant shift in $a_{\mathrm{w}}$ (black, dotted, lines) for leonardite (LEO, $\left.\Delta a_{\mathrm{w}, \text { het }}=0.2703\right)$ and Pahokee peat (PP, $\left.\Delta a_{\mathrm{w} \text {, het }}=0.2466\right)$. Red, blue and green colors correspond to $a_{\mathrm{W}}$ equal to $1.0,0.931$ and 0.872, respectively, for LEO, and 1.0, 0.901 and 0.862 for PP. Shaded area corresponds to $\Delta a_{\mathrm{w} \text {, het }} \pm 0.025$. Markers correspond to experimental measurements reported by Rigg et al. (2013); error bars represent an order of magnitude deviation from the reported value. (b): $J_{\text {het }}$ calculated for constant $\zeta=0.949$ for LEO and $\zeta=0.952$ for PP. The shaded area corresponds to $a_{\mathrm{W}} \pm 0.01$ and $\zeta \pm 0.0015$.

may fail for spinodal ice nucleation, since it does not consider the effect of the particle on $J_{0} . \Delta a_{\mathrm{w} \text {, het }}$ however carries important information about $J_{\text {het }}$ (Knopf and Alpert, 2013), but for spinodal ice nucleation, the relationship between $\Delta a_{\mathrm{w}, \text { het }}$ and $\zeta$ must be more complex than predicted by Eq. (69), since kinetic limitations play a significant role. Figure 8 also shows that similar $T_{\mathrm{f}}$ can be obtained by either high or low $\zeta$. The particular regime in which an INP nucleates ice determines $-\frac{\mathrm{d} \ln J_{\text {het }}}{\mathrm{d} T}$, hence the sensitivity of the droplet freezing rate to the particle size and to the cooling rate.

\subsection{Limitations}

It is important to analyze the effect of several assumptions introduced in Sect. 2 on the theory presented here. One of the limitations of the approach used in deriving Eq. (61) is that it employs macroscale thermodynamics in the formulation of the work of nucleation. The effect of this assumption is, however, minimized in several ways. First, unlike frameworks based on the interfacial tension, NNF is much more robust to changes in ice germ size, since the product $\Gamma_{\mathrm{w}} s \Delta h_{\mathrm{f}}$ remains constant (Sect. 2.4). Second, in the spinodal regime $\Delta G_{\text {het }}$ is independent of $n_{\text {het }}$, and only for $T>268 \mathrm{~K}$ and in the germ-forming regime, the approach presented here may lead to uncertainty (Sect. 3.1). Thus Eq. (61) remains 
valid for most atmospheric conditions, although caution must be taken when $T_{\mathrm{f}}>268 \mathrm{~K}$. Alternatively the framework presented here could be extended to account explicitly for the effect of size on $\Delta h_{\mathrm{f}}$ and $\Gamma_{\mathrm{w}}$ (e.g., Zhang et al., 1999).

Further improvement could be achieved by implementing a more sophisticated equation of state of the vicinal water. Here a two-state assumption has been used, such that $\mu_{\mathrm{vc}}$ is a linear combination of ice-like and liquid-like fractions. Such approximation has been used with success before (Etzler, 1983; Holten et al., 2013). However it is known that the structure of supercooled water represents an average of several distinct configurations (Stanley and Teixeira, 1980). These are, in principle, accounted for in the proposed approach, since $\zeta$ represents a relative, not an absolute, increase in the IL fraction. However there is no guarantee that such an increase can be linearly mapped in the way described in Sect. 2. Fortunately this would only mean, in practice, that the value of $\zeta$ for a given material is linked to the particular form of the equation of state used to describe the vicinal water.

Equation (61) is also blind to the surface properties of the immersed particle. The implicit assumption is that the effect of surface composition, charge, hydrophilicity and roughness on $J_{\text {het }}$ can be parameterized as a function of $\zeta$. The example shown in Sect. 3.4 suggests that this is indeed the case. Making such relations explicit must, however, lie at the center of future development of the proposed approach. Similarly, a heuristic approach was used to study the effect of irreversibility on the nucleation work. This can be improved substantially by making use of a generalized Gibbs approach (Schmelzer et al., 2006), which unfortunately may also increase the number of free parameters in the model. None of these limitations is expected to change the conclusions of this study, however they may affect the values of $\zeta$ fitted when analyzing experimental data. The approach proposed here, however, has the advantage of being a simple, one-parameter approximation that can be easily implemented in cloud models.

\section{Summary and conclusions}

Immersion freezing is a fundamental cloud process, and its correct representation in atmospheric models is critical for accurate climate and weather predictions. Current theories rely on a view that mimics ice formation from the vapor, neglecting several interactions unique to the liquid. This work develops, for the first time, a comprehensive approach to account for such interactions. The ice nucleation activity of immersed particles is linked to their effect on the vicinal water. It is shown that the same mechanism that lowers the thermodynamic barrier for ice nucleation also tends to decrease the mobility of water molecules, hence limiting interfacial transfer and ice germ growth. The role of the immersed particle in ice nucleation can be understood as increasing order in the adjacent water, facilitating the formation of ice-like structures. Thus, instead of being purely driven by thermodynamics, heterogeneous ice nucleation in the liquid phase is a process determined by the competition between thermodynamic and kinetic constraints to the formation and propagation of ice.

In the new approach the properties of vicinal water are approximated using a regular solution between high and lowdensity regions, with composition defined by an material specific parameter, $\zeta$, which acts as a "templating factor" for ice nucleation. This results on an identity between the homogeneous and the heterogeneous work of nucleation (Eq. 35), implying that by knowing an expression for $\Delta G_{\text {hom }}, \Delta G_{\text {het }}$ can be readily written. This is advantageous, as homogeneous ice nucleation is far better understood than immersion ice nucleation and, because it avoids a mechanistic description of the complex interaction between the particle, the ice and the liquid. To describe $\Delta G_{\text {hom }}$ the NNF framework (Barahona, 2014) was employed. This approach was extended to include non-equilibrium dissipation effects.

A model to describe the effect of the immersed particle on the mobility of water molecules, hence on the kinetics of immersion freezing, was also developed. This model builds upon an expression for the interfacial diffusion flux that accounts for the work required for water molecules to accommodate in an ice-like manner during interface transfer. Here this expression is extended to account for the effect of the particle on the molecular flux to the ice germ. It was shown that $J_{0}$ strongly decreases as the system moves towards thermodynamic equilibrium.

The model presented here suggests the existence of a spinodal regime in ice nucleation where a pair of molecules with orientation similar to that of bulk ice may be enough to trigger freezing. Ice nucleation in the spinodal regime requires a highly efficient templating effect by the particle, however also tends to be strongly limited by the kinetics of the ice-liquid interfacial transfer. Compared to the classical germ-forming regime, nucleation by a spinodal mechanism is much more limited by diffusion and exhibits a more moderate increase in $J_{\text {het }}$ as temperature decreases. The existence of two nucleation regimes and the strong kinetic limitations occurring in efficient INPs imply that the freezing temperature is an ambiguous measure of ice nucleation activity. This is because, for a given $T$, two INPs characterized by different $\zeta$ may have the same $J_{\text {het }}$, although with very different sensitivity to surface area and cooling rate.

The relationship between the measured shift in water activity $\Delta a_{\mathrm{w} \text {,het }}$ and $\zeta$ was analyzed. It was shown that the proposed model leads directly to the derivation of the so-called water activity criterion for heterogeneous ice nucleation. The concept of "thermodynamic freezing temperature" was introduced and defined as the highest temperature at which it is likely to observe ice nucleation for a given thermodynamic state. $T_{\mathrm{ft}}$ is useful in analyzing how changes in the thermody- 
namic environment around the droplet affect ice nucleation, independently of the freezing kinetics.

The theory presented here was tested using data for humiclike substances. It was found that assuming a fixed water activity shift to predict $J_{\text {het }}$ could be appropriate for low $\zeta$, as found in leonardite (the germ-forming regime); however this may lead to overprediction of $-\frac{\mathrm{d} \ln J_{\text {het }}}{\mathrm{d} T}$ for the high $\zeta$ characterizing Pahokee peat INPs. This is because the water activity criterion represents a thermodynamic relation between $a_{\mathrm{w}}$ and $T_{\mathrm{f}}$ but does not account for kinetic limitations, which may be significant in spinodal ice nucleation.

Immersion freezing research has seen a resurgence during the last decade (DeMott et al., 2011). A wealth of data is now available to test theories and new approaches to describe ice formation in atmospheric models. To do so effectively, it is necessary to develop models that realistically capture the complexities of the liquid phase. Further development of the approach presented here will look to better describe the nonreversible aspects of nucleation as well as to establish a more complete description of the properties of the vicinal water. The application to the freezing of atmospheric aerosol requires the definition of the ice nucleation spectrum, which will be pursued in a future work. Nevertheless, the present study constitutes, for first the time, an approximation to the modeling of ice nucleation that links the modifications of the properties of vicinal water by immersed particles with their ice nucleation ability. The approach presented here may help with expanding our understanding of immersion ice nucleation and facilitating the interpretation of experimental data in situations where current models fall short. The application of these ideas in cloud models will allow for the elucidation of the conditions under which different nucleation regimes occur in the atmosphere.

Data availability. Supplementary material includes data used in generating Figs. 4-6. Fortran subroutines with the full implementation of the theory are available upon request. 


\section{Appendix A: List of symbols.}

$a_{0} \quad$ Cross-sectional area of a water molecule, $\pi d_{0}^{2} / 4, \mathrm{~m}^{2}$

$A_{\mathrm{w}} \quad$ Phenomenological interaction parameter

$a_{\mathrm{w}} \quad$ Activity of water

$a_{\mathrm{w}, \mathrm{eff}} \quad$ Effective water activity

$a_{\mathrm{w}, \mathrm{eq}}$

Equilibrium $a_{\mathrm{w}}$ between bulk liquid and ice (Koop and Zobrist, 2009)

$a_{\mathrm{w}, \text { het }}$

$a_{\mathrm{w}, \mathrm{hom}}$

$C_{0}$

$E, T_{0}$

$D$

Thermodynamic freezing threshold for heterogeneous ice nucleation

Thermodynamic freezing threshold for homogeneous ice nucleation

Monomer concentration, $\mathrm{m}^{-2}$

Parameters of the Vogel-Fulcher-Tammann equation defining $D_{\infty}, 892$ and $118 \mathrm{~K}$ (Smith and Kay, 1999)

Diffusion coefficient for interface transfer, $\mathrm{m}^{2} \mathrm{~s}^{-1}$

Self-diffusion coefficient of bulk water (Smith and Kay, 1999), $\mathrm{m}^{2} \mathrm{~s}^{-1}$

$D_{0} \quad$ Fitting parameter, $3.06 \times 10^{-9} \mathrm{~m}^{2} \mathrm{~s}^{-1}$ (Smith and Kay, 1999)

$d_{0} \quad$ Molecular diameter of water, $\left(6 v_{\mathrm{w}} / \pi\right)^{1 / 3}, \mathrm{~m}$

$f_{\text {het }}^{*} \quad$ Impingement factor for heterogeneous ice nucleation, $\mathrm{s}^{-1}$

$f_{\text {hom }}^{*} \quad$ Impingement factor for homogeneous ice nucleation, $\mathrm{s}^{-1}$

$G \quad$ Gibbs free energy, J

$h \quad$ Planck's constant, J s

$J_{0} \quad$ Pre-exponential factor $\mathrm{m}^{-2} \mathrm{~s}^{-1}$

$J_{\text {het }} \quad$ Heterogeneous nucleation rate, $\mathrm{m}^{-2} \mathrm{~s}^{-1}$

$k_{B} \quad$ Boltzmann constant, $\mathrm{JK}^{-1}$

$N \quad$ Number of clustering molecules in LL and IL regions, 6 (Holten et al., 2013)

$n \quad$ Number of molecules in an ice cluster

$n^{*} \quad$ Critical germ size

$n_{\text {het }} \quad$ Critical germ size for heterogeneous ice nucleation

$n_{\text {hom }} \quad$ Critical germ size for homogeneous ice nucleation

$n_{\mathrm{t}}$

$p_{\mathrm{s}, \mathrm{w}}, p_{\mathrm{s}, \mathrm{i}} \quad$ Liquid water and ice saturation vapor pressure, respectively, Pa(Murphy and Koop, 2005)

$s \quad$ Geometric constant of the ice lattice, 1.105 molec $^{1 / 3}$ (Barahona, 2014)

$S_{\mathrm{i}} \quad$ Saturation ratio with respect to ice

$S_{\mathrm{c}, 0} \quad$ Configuration entropy of water*

$S_{\mathrm{c}} \quad$ Configuration entropy of vicinal water

$T \quad$ Temperature, $\mathrm{K}$

$T_{\mathrm{c}} \quad$ Critical separation temperature, $211.473 \mathrm{~K}$

$v_{\mathrm{w}} \quad$ Molecular volume of water in ice (Zobrist et al., 2007), $\mathrm{m}^{-3}$

$v_{\mathrm{w}, 0} \quad$ Molecular volume of water at $273.15 \mathrm{~K}$

$\bar{W} \quad$ Average transition probability in water

$W_{\text {diss }} \quad$ Work dissipated during cluster formation, $\mathrm{J}$

$W_{\mathrm{d}} \quad$ Work dissipated during interface transfer, $\mathrm{J}$

Z Zeldovich factor

$\Delta a_{\mathrm{w}, \text { het }} \quad a_{\mathrm{w}, \text { het }}-a_{\mathrm{w}, \mathrm{eq}}$

$\Delta a_{\mathrm{w}, \text { hom }} \quad a_{\mathrm{w}, \mathrm{hom}}-a_{\mathrm{w}, \mathrm{eq}}, 0.304$ (Koop et al., 2000; Barahona, 2014)

$\Delta G \quad$ Work of cluster formation, $\mathrm{J}$

$\Delta G_{\text {act }} \quad$ Activation energy for ice nucleation, $\mathrm{J}$

$\Delta G_{\text {hom }} \quad$ Nucleation work for homogeneous ice nucleation, $\mathrm{J}$

$\Delta G_{\text {het }} \quad$ Nucleation work for heterogeneous ice nucleation, J

$\Delta h_{\mathrm{f}} \quad$ Heat of solidification of water, $\mathrm{J} \mathrm{mol}^{-1}$ (Barahona et al., 2014; Johari et al., 1994)

$\Delta \mu_{\mathrm{s}} \quad$ Excess free energy of solidification of water, $\mathrm{J}$

$\Delta \mu_{\mathrm{i}} \quad$ Driving force for ice nucleation, $\mathrm{J}$

$\Lambda_{\text {mix }} \quad$ Dimensionless mixing parameter, defined in Eq. (30)

$\Phi \quad$ Energy of formation of the ice-liquid interface, molec $^{1 / 3} \mathrm{~J}$

$\Gamma_{\mathrm{w}} \quad$ Molecular surface excess at the interface, 1.46 (Barahona et al., 2014; Spaepen, 1975)

$\mu_{\mathrm{w}}, \mu_{\mathrm{s}}, \mu_{\mathrm{vc}} \quad$ Chemical potential of water, ice and vicinal water, respectively $\mathrm{J}$

$\rho_{\mathrm{w}}, \rho_{\mathrm{i}} \quad$ Bulk density of liquid water and ice, respectively, $\mathrm{Kg} \mathrm{m}^{-3}$ (Pruppacher and Klett, 1997)

$\sigma_{\mathrm{E}} \quad$ Dimensionless residual entropy

$\sigma_{\text {iw }} \quad$ Ice-liquid interfacial energy $\mathrm{J} \mathrm{m}^{-2}$ (Barahona et al., 2014)

$\theta \quad$ Contact angle

$\zeta \quad$ Templating factor

$\Omega_{\mathrm{g}} \quad$ Ice germ surface area, $\mathrm{m}^{-2}$

${ }^{*}$ From the data of Scala et al. (2000), the following fit was obtained: $S_{\mathrm{c}, 0}=k_{B} v_{\mathrm{w}} / v_{\mathrm{w}, 0}\left(-7.7481 \times 10^{-5} T^{2}+5.5160 \times 10^{-2} T-6.6716\right)\left(\mathrm{JK} \mathrm{K}^{-1}\right)$ for $T$ between 180 and $273 \mathrm{~K}$. 
Supplement. The supplement related to this article is available online at: https://doi.org/10.5194/acp-18-17119-2018-supplement.

Competing interests. The author declares that there is no conflict of interest.

Acknowledgements. Donifan Barahona was supported by the NASA Modeling and Analysis Program, grant: 16-MAP16-0085.

Edited by: Martina Krämer

Reviewed by: three anonymous referees

\section{References}

Adam, G. and Gibbs, J. H.: On the temperature dependence of cooperative relaxation properties in glass-forming liquids, J. Chem. Phys., 43, 139-146, 1965.

Alpert, P. A., Aller, J. Y., and Knopf, D. A.: Ice nucleation from aqueous $\mathrm{NaCl}$ droplets with and without marine diatoms, Atmos. Chem. Phys., 11, 5539-5555, https://doi.org/10.5194/acp11-5539-2011, 2011.

Anderson, D. M.: Ice nucleation and the substrate-ice interface, Nature, 216, 563-566, https://doi.org/10.1038/216563a0, 1967.

Atkinson, J. D., Murray, B. J., Woodhouse, M. T., Whale, T. F., Baustian, K. J., Carslaw, K. S., Dobbie, S., O'sullivan, D., and Malkin, T. L.: The importance of feldspar for ice nucleation by mineral dust inmixed-phase clouds, Nature, 498, 355-358, https://doi.org/10.1038/nature12278, 2013.

Baker, M. and Baker, M.: A new look at homogeneous freezing of water, Geophys. Res. Lett., 31, L19102, https://doi.org/10.1029/2004GL020483, 2004.

Barahona, D.: Analysis of the effect of water activity on ice formation using a new thermodynamic framework, Atmos. Chem. Phys., 14, 7665-7680, https://doi.org/10.5194/acp-147665-2014, 2014.

Barahona, D.: Thermodynamic derivation of the activation energy for ice nucleation, Atmos. Chem. Phys., 15, 13819-13831, https://doi.org/10.5194/acp-15-13819-2015, 2015.

Barahona, D. and Nenes, A.: Parameterization of cirrus formation in large scale models: Homogeneous nucleation, J. Geophys. Res., 113, D11211, https://doi.org/10.1029/2007JD009355, 2008.

Barahona, D. and Nenes, A.: Parameterizing the competition between homogeneous and heterogeneous freezing in cirrus cloud formation - monodisperse ice nuclei, Atmos. Chem. Phys., 9, 369-381, https://doi.org/10.5194/acp-9-369-2009, 2009.

Barahona, D., Molod, A., Bacmeister, J., Nenes, A., Gettelman, A., Morrison, H., Phillips, V., and Eichmann, A.: Development of two-moment cloud microphysics for liquid and ice within the NASA Goddard Earth Observing System Model (GEOS-5), Geosci. Model Dev., 7, 1733-1766, https://doi.org/10.5194/gmd7-1733-2014, 2014.

Barahona, D., Molod, A., and Kalesse, H.: Direct estimation of the global distribution of vertical velocity within cirrus clouds, Sci. Rep. UK, 7, 6840, https://doi.org/10.1038/s41598-017-07038-6, 2017.
Bellissent-Funel, M.-C.: Water near hydrophilic surfaces, J. Mol. Liquids, 96, 287-304, https://doi.org/10.1016/S01677322(01)00354-3, 2002.

Bigg, E. K.: The formation of atmospheric ice crystals by the freezing of droplets, Q. J. Roy. Meteor. Soc., 79, 510-519, https://doi.org/10.1002/qj.49707934207, 1953.

Black, S.: Simulating nucleation of molecular solids, P. R. Soc. A, 463, 2799-2811, 2007.

Broadley, S. L., Murray, B. J., Herbert, R. J., Atkinson, J. D., Dobbie, S., Malkin, T. L., Condliffe, E., and Neve, L.: Immersion mode heterogeneous ice nucleation by an illite rich powder representative of atmospheric mineral dust, Atmos. Chem. Phys., 12, 287-307, https://doi.org/10.5194/acp-12-287-2012, 2012.

Brukhno, A. V., Anwar, J., Davidchack, R., and Handel, R.: Challenges in molecular simulation of homogeneous ice nucleation, J. Phys.-Condens. Mat., 20, 494243, https://doi.org/10.1088/09538984/20/49/494243, 2008.

Bullock, G. and Molinero, V.: Low-density liquid water is the mother of ice: on the relation between mesostructure, thermodynamics and ice crystallization in solutions, Faraday Discuss., https://doi.org/10.1039/C3FD00085K, 2013.

Cahn, J. W.: Surface stress and the chemical equilibrium of small crystals-I. The case of the isotropic surface, Acta Metall. Mater, 28, 1333-1338, 1980.

Cahn, J. W. and Hilliard, J. E.: Free energy of a nonuniform system. I. Interfacial free energy, J. Chem. Phys., 28, 258-267, 1958.

Cooke, R. and Kuntz, I.: The properties of water in biological systems, Ann. Review Biophys. Bioeng., 3, 95-126, 1974.

Cox, S. J., Kathmann, S. M., Slater, B., and Michaelides, A.: Molecular simulations of heterogeneous ice nucleation. I. Controlling ice nucleation through surface hydrophilicity, J. Chem. Phys, 142, 184704, https://doi.org/10.1063/1.4919714, 2015.

Cziczo, D. J., Froyd, K. D., Hoose, C., Jensen, E. J., Diao, M., Zondlo, M. A., Smith, J. B., Twohy, C. H., and Murphy, D. M.: Clarifying the Dominant Sources and Mechanisms of Cirrus Cloud Formation, Science, 340, 1320-1324, 2013.

De Gennes, P.-G.: Wetting: statics and dynamics, Rev. Modern Phys., 57, 827-863, https://doi.org/10.1103/RevModPhys.57.827, 1985.

Debenedetti, P. G. and Stillinger, F. H.: Supercooled liquids and the glass transition, Nature, 410, 259-267, 2001.

DeMott, P., Cziczo, D., Prenni, A., Murphy, D., Kreidenweis, S., Thompson, D., Borys, R., and Rogers, D.: Measurements of the concentration and composition of nuclei for cirrus formation, Proc. Natl. Acad. Sci. USA, 100, 14655-14660, 2003.

DeMott, P. J., Prenni, A. J., Liu, X., Kreidenweis, S. M., Petters, M. D., Twohy, C. H., Richardson, M. S., Eidhammer, T., and Rogers, D. C.: Predicting global atmospheric ice nuclei distributions and their impacts on climate, Proc. Natl. Acad. Sci. USA, 107, 11217-11222, https://doi.org/10.1073/pnas.0910818107, 2010.

DeMott, P. J., Möhler, O., Stetzer, O., Vali, G., Levin, Z., Petters, M. D., Murakami, M., Leisner, T., Bundke, U., Klein, H., et al.: Resurgence in ice nuclei measurement research, B. Am. Meteorol. Soc., 92, 1623-1635, 2011.

Diehl, K. and Wurzler, S.: Heterogeneous drop freezing in the immersion mode: model calculations considering soluble and insoluble particles in the drops, J. Atmos. Sci., 61, 2063-2072, 2004. 
Drost-Hansen, W.: Structure of water near solid interfaces, Ind. \& Eng. Chem., 61, 10-47, 1969.

Espinosa, J., Sanz, E., Valeriani, C., and Vega, C.: Homogeneous ice nucleation evaluated for several water models, J. Chem. Phys., 141, 18C529, https://doi.org/10.1063/1.4897524, 2014.

Etzler, F. M.: A statistical thermodynamic model for water near solid interfaces, J. Coll. Interf. Sci., 92, 43-56, 1983.

Feibelman, P. J.: The first wetting layer on a solid, Physics today, 63, 34, https://doi.org/10.1063/1.3326987, 2010.

Fitzner, M., Sosso, G. C., Cox, S. J., and Michaelides, A.: The Many Faces of Heterogeneous Ice Nucleation: Interplay Between Surface Morphology and Hydrophobicity, J. Am. Chem. Soc., 137, 13658-13669, https://doi.org/10.1021/jacs.5b08748, 2015.

Fletcher, H.: On ice-crystal production by aerosol particles, J. Atmos. Sci., 16, 173-180, 1959.

Gettelman, A., Liu, X., Barahona, D., Lohmann, U., and Chen, C.: Climate impacts of ice nucleation, J. Geophys. Res., 117, D20201, https://doi.org/10.1029/2012JD017950, 2012.

Hiranuma, N., Augustin-Bauditz, S., Bingemer, H., Budke, C., Curtius, J., Danielczok, A., Diehl, K., Dreischmeier, K., Ebert, M., Frank, F., Hoffmann, N., Kandler, K., Kiselev, A., Koop, T., Leisner, T., Möhler, O., Nillius, B., Peckhaus, A., Rose, D., Weinbruch, S., Wex, H., Boose, Y., DeMott, P. J., Hader, J. D., Hill, T. C. J., Kanji, Z. A., Kulkarni, G., Levin, E. J. T., McCluskey, C. S., Murakami, M., Murray, B. J., Niedermeier, D., Petters, M. D., O’Sullivan, D., Saito, A., Schill, G. P., Tajiri, T., Tolbert, M. A., Welti, A., Whale, T. F., Wright, T. P., and Yamashita, K.: A comprehensive laboratory study on the immersion freezing behavior of illite NX particles: a comparison of 17 ice nucleation measurement techniques, Atmos. Chem. Phys., 15, 2489-2518, https://doi.org/10.5194/acp-15-2489-2015, 2015.

Holten, V., Limmer, D. T., Molinero, V., and Anisimov, M. A.: Nature of the anomalies in the supercooled liquid state of the $\mathrm{mW}$ model of water, J. Chem. Phys., 138, 174501, https://doi.org/10.1063/1.4802992, 2013.

Hoose, C. and Möhler, O.: Heterogeneous ice nucleation on atmospheric aerosols: a review of results from laboratory experiments, Atmos. Chem. Phys., 12, 9817-9854, https://doi.org/10.5194/acp-12-9817-2012, 2012.

Hoose, C., Kristjansson, J., Chen, J.-C., and Hazra, A.: A classical-theory-based parameterization of heterogeneous ice nucleation by mineral dust, soot, and biological particles in a global climate model, J. Atmos. Sci., 67, 2483-2503, https://doi.org/10.1175/2010JAS3425.1, 2010.

Ickes, L., Welti, A., and Lohmann, U.: Classical nucleation theory of immersion freezing: sensitivity of contact angle schemes to thermodynamic and kinetic parameters, Atmos. Chem. Phys., 17, 1713-1739, https://doi.org/10.5194/acp-17-1713-2017, 2017.

Johari, G., Fleissner, G., Hallbrucker, A., and Mayer, E.: Thermodynamic continuity between glassy and normal water, J. Phys. Chem., 98, 4719-4725, 1994.

Johnston, J. C. and Molinero, V.: Crystallization, melting, and structure of water nanoparticles at atmospherically relevant temperatures, JACS, 134, 6650-6659, https://doi.org/10.1021/ja210878c, 2012.

Kalikmanov, V. I. and van Dongen, M. E. H.: Selfconsistent cluster approach to the homogeneous kinetic nucleation theory, Phys. Rev. E, 47, 3532-3539, https://doi.org/10.1103/PhysRevE.47.3532, 1993.
Kärcher, B. and Lohmann, U.: A parameterization of cirrus cloud formation: Heterogeneous freezing, J. Geophys. Res., 108, 4402, https://doi.org/10.1029/2002JD003220, 2003.

Kashchiev, D.: Nucleation: basic theory with applications, Butterworth Heinemann, Burlington, MA, USA, 2000.

Khvorostyanov, V. and Curry, J.: The theory of ice nucleation by heterogeneous freezing of deliquescent mixed CCN. Part I: critical radius, energy and nucleation rate, J. Atmos. Sci., 61, 26762691, 2004.

Khvorostyanov, V. and Curry, J.: The theory of ice nucleation by heterogeneous freezing of deliquescent mixed CCN. Part II: parcel model simulations, J. Atmos. Sci., 62, 261-285, 2005.

Kiselev, A., Bachmann, F., Pedevilla, P., Cox, S. J., Michaelides, A., Gerthsen, D., and Leisner, T.: Active sites in heterogeneous ice nucleation - the example of K-rich feldspars, Science, 355, 367-371, https://doi.org/10.1126/science.aai8034, 2017.

Knopf, D. A. and Alpert, P. A.: A water activity based model of heterogeneous ice nucleation kinetics for freezing of water and aqueous solution droplets, Faraday Discuss., 165, 513-534, 2013.

Koop, T. and Zobrist, B.: Parameterizations for ice nucleation in biological and atmospheric systems, Phys. Chem. Chem. Phys., 11, 10839-10850, 2009.

Koop, T., Luo, B., Tslas, A., and Peter, T.: Water activity as the determinant for homogeneous ice nucleation in aqueous solutions, Nature, 406, 611-614, 2000.

Lance, S., Shupe, M. D., Feingold, G., Brock, C. A., Cozic, J., Holloway, J. S., Moore, R. H., Nenes, A., Schwarz, J. P., Spackman, J. R., Froyd, K. D., Murphy, D. M., Brioude, J., Cooper, O. R., Stohl, A., and Burkhart, J. F.: Cloud condensation nuclei as a modulator of ice processes in Arctic mixed-phase clouds, Atmos. Chem. Phys., 11, 8003-8015, https://doi.org/10.5194/acp11-8003-2011, 2011.

Li, K., Xu, S., Chen, J., Zhang, Q., Zhang, Y., Cui, D., Zhou, X., Wang, J., and Song, Y.: Viscosity of interfacial water regulates ice nucleation, App. Phys. Lett., 104, 101605, https://doi.org/10.1063/1.4868255, 2014.

Lohmann, U. and Feichter, J.: Global indirect aerosol effects: a review, Atmos. Chem. Phys., 5, 715-737, https://doi.org/10.5194/acp-5-715-2005, 2005.

Lupi, L., Hudait, A., and Molinero, V.: Heterogeneous Nucleation of Ice on Carbon Surfaces, J. Am. Chem. Soc., 136, 3156-3164, 2014.

Marcolli, C., Gedamke, S., Peter, T., and Zobrist, B.: Efficiency of immersion mode ice nucleation on surrogates of mineral dust, Atmos. Chem. Phys., 7, 5081-5091, https://doi.org/10.5194/acp7-5081-2007, 2007.

Matsumoto, M., Saito, S., and Ohmine, I.: Molecular dynamics simulation of the ice nucleation and growth process leading to water freezing, Nature, 416, 409-413, 2002.

Meyers, M., DeMott, P., and Cotton, R.: New primary icenucleation parameterization in an explicit cloud model, J. Appl. Meteorol., 31, 708-721, 1992.

Michaelides, A. and Morgenstern, K.: Ice nanoclusters at hydrophobic metal surfaces, Nat. Mater., 6, 597-601, https://doi.org/10.1038/nmat1940, 2007.

Michot, L. J., Villiéras, F., François, M., Bihannic, I., Pelletier, M., and Cases, J.-M.: Water organisation at the solid-aqueous solution interface, C. R. Geosci., 334, 611-631, 2002. 
Murphy, D. and Koop, T.: Review of the vapour pressures of ice and supercooled water for atmospheric applications, Q. J. Roy. Meteor. Soc., 131, 1539-1565, 2005.

Murray, B. J., O'sullivan, D., Atkinson, J. D., and Webb, M. E.: Ice nucleation by particles immersed in supercooled cloud droplets, Chem. Soc. Rev., 41, 6519-6554, https://doi.org/10.1039/C2CS35200A, 2012.

Myhre, G., Shindell, D., Breon, F.-M., Collins, W., Fuglestvedt, J., Huang, J., Koch, D., Lamarque, J.-F., Lee, D., Mendoza, B., Nakajima, T., Robock, A., Stephens, G., Takemura, T., and Zhang, H.: Anthropogenic and Natural Radiative Forcing. Climate Change 2013: The Physical Science Basis. Contribution of Working Group I to the Fifth Assessment Report of the Intergovernmental Panel on Climate Change, book section 8, 659-740, Cambridge University Press, Cambridge, United Kingdom and New York, NY, USA, https://doi.org/10.1017/CBO9781107415324.018, 2013.

Niemand, M., Möhler, O., Vogel, B., Vogel, H., Hoose, C., Connolly, P., Klein, H., Bingemer, H., DeMott, P., Skrotzki, J., and Leisner, T.: A particle-surface-area-based parameterization of immersion freezing on desert dust particles, J. Atmos. Sci., 69, 3077-3092, https://doi.org/10.1175/JAS-D-11-0249.1, 2012.

O, K.-T. and Wood, R.: Exploring an approximation for the homogeneous freezing temperature of water droplets, Atmos. Chem. Phys., 16, 7239-7249, https://doi.org/10.5194/acp-167239-2016, 2016.

Pauling, L.: The structure and entropy of ice and of other crystals with some randomness of atomic arrangement, J. Am. Chem. Soc., 57, 2680-2684, 1935.

Phillips, V. T., Demott, P. J., Andronache, C., Pratt, K. A., Prather, K. A., Subramanian, R., and Twohy, C.: Improvements to an Empirical Parameterization of Heterogeneous Ice Nucleation and its Comparison with Observations, J. Atmos. Sci., 70, 378-409, https://doi.org/10.1175/JAS-D-12-080.1, 2013.

Prausnitz, J. M., Lichtenthaler, R. N., and de Azevedo, E. G.: Molecular thermodynamics of fluid-phase equilibria, Prentice Hall, Upper Saddle River, NJ, USA, 3rd edn., 1998.

Pruppacher, H. and Klett, J.: Microphysics of clouds and precipitation, Kluwer Academic Publishers, Boston, MA, 2nd edn., 1997.

Rigg, Y. J., Alpert, P. A., and Knopf, D. A.: Immersion freezing of water and aqueous ammonium sulfate droplets initiated by humic-like substances as a function of water activity, Atmos. Chem. Phys., 13, 6603-6622, https://doi.org/10.5194/acp13-6603-2013, 2013.

Rinnert, E., Carteret, C., Humbert, B., Fragneto-Cusani, G., Ramsay, J. D., Delville, A., Robert, J.-L., Bihannic, I., Pelletier, M., and Michot, L. J.: Hydration of a synthetic clay with tetrahedral charges: a multidisciplinary experimental and numerical study, J. Phys. Chem. B, 109, 23745-23759, 2005.

Rusanov, A. I.: Surface thermodynamics revisited, Surf. Sc. Reports, 58, 111-239, 2005.

Scala, A., Starr, F. W., La Nave, E., Sciortino, F., and Stanley, H. E.: Configurational entropy and diffusivity of supercooled water, Nature, 406, 166-169, 2000.

Schmelzer, J. W., Boltachev, G. S., and Baidakov, V. G.: Classical and generalized Gibbs' approaches and the work of critical cluster formation in nucleation theory, J. Chem. Phys., 124, 194503, https://doi.org/10.1063/1.2196412, 2006.
Seinfeld, J. H. and Pandis, S. N.: Atmospheric Chemistry and Physics, John Wiley and Sons, New York, NY, USA, 1998.

Smith, R. S. and Kay, B. D.: The existence of supercooled liquid water at $150 \mathrm{~K}$, Nature, 398, 788-791, 1999.

Snyder, P. W., Lockett, M. R., Moustakas, D. T., and Whitesides, G. M.: Is it the shape of the cavity, or the shape of the water in the cavity?, The European Physical Journal Special Topics, 223, 853-891, https://doi.org/10.1140/epjst/e2013-01818-y, 2014.

Spaepen, F.: A structural model for the solid-liquid interface in monatomic systems, Acta Metall. Mater, 23, 729-743, 1975.

Stanley, H. E. and Teixeira, J.: Interpretation of the unusual behavior of $\mathrm{H} 2 \mathrm{O}$ and $\mathrm{D} 2 \mathrm{O}$ at low temperatures: tests of a percolation model, J. Chem. Phys., 73, 3404-3422, 1980.

Tan, I., Storelvmo, T., and Zelinka, M. D.: Observational constraints on mixed-phase clouds imply higher climate sensitivity, Science, 352, 224-227, https://doi.org/10.1126/science.aad5300, 2016.

Taylor, J. H. and Hale, B. N.: Monte Carlo simulations of waterice layers on a model silver iodide substrate: A comparison with bulk ice systems, Physical Rev. B, 47, 9732, 1993.

Turnbull, D. and Fisher, J. C.: Rate of nucleation in condensed systems, J. Chem. Phys., 17, 71-73, 1949.

Vali, G.: Interpretation of freezing nucleation experiments: singular and stochastic; sites and surfaces, Atmos. Chem. Phys., 14, 5271-5294, https://doi.org/10.5194/acp-14-5271-2014, 2014.

Vekilov, P. G.: The two-step mechanism of nucleation of crystals in solution, Nanoscale, 2, 2346-2357, https://doi.org/10.1039/C0NR00628A, 2010.

Wang, J., Kalinichev, A. G., and Kirkpatrick, R. J.: Effects of substrate structure and composition on the structure, dynamics, and energetics of water at mineral surfaces: A molecular dynamics modeling study, Geochim. Cosmochim. Ac., 70, 562-582, https://doi.org/10.1016/j.gca.2005.10.006, 2006.

Wang, Q., Zhao, L., Li, C., and Cao, Z.: The decisive role of free water in determining homogenous ice nucleation behavior of aqueous solutions, Sci. Rep. UK, 6, 26831, https://doi.org/10.1038/srep26831, 2016.

Warne, M., Allan, N., and Cosgrove, T.: Computer simulation of water molecules at kaolinite and silica surfaces, Phys. Chem. Chem. Phys., 2, 3663-3668, 2000.

Wiacek, A., Peter, T., and Lohmann, U.: The potential influence of Asian and African mineral dust on ice, mixed-phase and liquid water clouds, Atmos. Chem. Phys., 10, 8649-8667, https://doi.org/10.5194/acp-10-8649-2010, 2010.

Wolfe, J., Bryant, G., and Koster, K. L.: What is' unfreezable water', how unfreezable is it and how much is there?, CryoLetters, 23, 157-166, 2002.

Yu, C.-J., Evmenenko, G., Richter, A., Datta, A., Kmetko, J., and Dutta, P.: Order in molecular liquids near solid-liquid interfaces, App. Surface Sci., 182, 231-235, https://doi.org/10.1016/S01694332(01)00410-X, 2001.

Zhang, Z., Lü, X., and Jiang, Q.: Finite size effect on melting enthalpy and melting entropy of nanocrystals, Physica B, 270, 249254, https://doi.org/10.1016/S0921-4526(99)00199-4, 1999.

Zheng, J.-m., Chin, W.-C., Khijniak, E., Khijniak Jr, E., and Pollack, G. H.: Surfaces and interfacial water: evidence that hydrophilic surfaces have long-range impact, Adv. Colloid Interface Sc., 127, 19-27, https://doi.org/10.1016/j.cis.2006.07.002, 2006.

Zobrist, B., Koop, T., Luo, B., Marcolli, C., and Peter, T.: Heterogeneous ice nucleation rate coefficient of water droplets coated 
by a nonadecanol monolayer, J. Phys. Chem. C, 111, 2149-2155, 2007.

Zobrist, B., Marcolli, C., Peter, T., and Koop, T.: Heterogeneous ice nucleation in aqueous solutions: the role of water activity, J. Phys. Chem. A, 112, 3965-3975, 2008.
Zuberi, B., Bertram, A., Cassa, C., Molina, L., and Molina, M. Heterogeneous nucleation of ice in $\left(\mathrm{NH}_{4}\right)_{2} \mathrm{SO}_{4}-\mathrm{H}_{2} \mathrm{O}$ particles with mineral dust immersions, Geophys. Res. Lett., 29, 1504, https://doi.org/10.1029/2001GL014289, 2002. 